\title{
Equilibrium Existence and Approximation for Incomplete Market Models with Substantial Heterogeneity*
}

\author{
Thomas M. Mertens ${ }^{\dagger} \quad$ Kenneth L. Judd ${ }^{\ddagger}$
}

November 26, 2012

\begin{abstract}
This paper contains an analysis of incomplete market models with finitely but arbitrarily many heterogeneous agents. We discuss the mathematical foundation for equilibrium conditions which leads to two findings. First, we establish existence of equilibria for small and large risks. Second, we develop a simple but general solution technique which handles many state and choice variables for each agent and thus an extremely high-dimensional state space. The method is based on perturbations around a point at which the solution is known. The novel idea is to exploit the symmetry of the problem to overcome the curse of dimensionality. We use the analysis to demonstrate the impact of heterogeneity on macroeconomic quantities and the pricing of risk. Furthermore, we set our technique apart from the standard method used in the literature.
\end{abstract}

\footnotetext{
*We thank Marios Angeletos, Dave Backus, Fernando Duarte, Darrell Duffie, Xavier Gabaix, John Geanakoplos, Sydney Ludvigson, Hanno Lustig, Semyon Malamud, Marti Subrahmanyam, Stijn van Nieuwerburgh, Kjetil Storesletten, and the audiences at the New York Fed, NYU Stern, Wharton, Duke, 2011 SCE conference, SITE 2011, the Chicago Booth-Deutsche Bank symposium, the Cowles GE conference, the SED, and the EFA for helpful comments. An earlier version of the paper was circulated under the title "Solving General Incomplete Market Models with Substantial Heterogeneity"

${ }^{\dagger}$ New York University, Stern School of Business; Postal Address: 44 W Fourth Street, Suite 9-73, New York, NY 10012, USA; E-mail: mertens@stern.nyu.edu.

${ }^{\ddagger}$ Hoover Institution, Stanford University; Postal Address: 434 Galvez Mall, Stanford, CA 94305, USA; E-mail: judd@hoover.stanford.edu
} 


\section{Introduction}

A large body of literature in finance and macroeconomics makes the simplifying assumption that aggregate variables are determined by the behavior of a representative agent. In reality, different people earn different incomes, have different talents, and hold different expectations. For this heterogeneity to be reflected in economic outcomes, incompleteness of asset markets is essential. In reality, substantial amounts of idiosyncratic risk can only be partially insured. Labor income risk serves as one of the prime examples. Modeling this type of idiosyncratic risk permits a more stringent test of our current economic theory since we can use information about the entire distribution of economic variables across the population.

This paper provides a formal analysis of a broad range of incomplete markets models with substantial heterogeneity, i.e. an economy with finitely but arbitrarily many different agents. This analysis leads to two findings. First, we prove existence of equilibria for an incomplete markets economy. We discuss the relevant theory for local and global existence to make the technique for proofs as portable as possible. Second, we find a simple but general solution method for economies in which the state space is very large. A multitude of state variables arises from heterogeneity but might increase if there are several variables for each individual.

As a leading example of the paper, we analyze a dynamic stochastic general equilibrium model with aggregate risk in production and an endogenous capital stock. A firm produces a single consumption good which households consume according to a Cobb-Douglas production function. Future total factor productivity is uncertain due to aggregate shocks. Households maximize expected discounted utility given by a utility function featuring constant relative risk aversion in consumption. We add a cost of deviating from a target level of capital. This cost serves two purposes. First, it makes borrowing costly and thus serves as an endogenous borrowing constraint. Second, it facilitates the solution method as it determines the distribution of capital in the deterministic steady-state. Given their utility function and budget constraint, each household decides how much to consume and save each period.

We add idiosyncratic shocks to labor income which agents cannot insure against. Households only trade claims to capital which renders markets incomplete. As a result, equilibrium outcomes feature idiosyncratic components. Households hold different levels of capital which translates into inequality of wealth and consumption.

The analysis of this model presents a difficult problem. Ultimately, we want to be able to study the interaction of choices and prices with the distribution of assets. In particular, we need a solution method that solves for individual behavior and aggregate variables including asset prices as a function of the entire distribution of economic conditions. But, in turn, this 
distribution is affected by all individuals' behavior. In other words, the state space might contain several distributions of variables across the population.

We lay out the mathematical structure of equilibrium conditions. In the case where we eliminate all uncertainty, the optimality conditions define the solution to the deterministic economy, a collection of consumption and investment functions and prices. We discuss the properties of the equilibrium operator which is comprised of the equilibrium conditions. It maps functions from a Banach space to a Banach space where the equilibrium operator itsself is differentiable. We discuss the appropriate notion of Freéchet differentiability in detail. Differentiability is a key aspect of the operator since we want to apply methods from nonlinear functional analysis.

We show how to use this mathematical foundation to establish existence of equilibria. We demonstrate two different sets of results for our example economy. First, we establish local existence, i.e. the case of small risk, and uniqueness. We obtain these implications from the Implicit Function Theorem for operators in Banach spaces. Second, we establish existence for the economy with large risks as well. The proof is based on the Leray-Schauder Continuation Theorem which extends the range of the Implicit Function Theorem. This theorem lays out sufficient conditions under which the local solution can be extended once we increase the risk in the economy.

To compute the equilibrium of our economy, we develop a solution technique for models with many heterogeneous agents and incomplete markets based on perturbation methods. Perturbation methods build an approximation of the optimal policies as functions of the state variables based on Taylor expansions. The first step is to find a special case of the model in which the solution is known. Our model possesses a well-defined deterministic steady-state around which we expand optimal policies with respect to all state variables. At the point of expansion, all agents are identical in all respects and thus we have a degenerate distribution of capital. Having pinned down the deterministic steady-state, we build an expansion with respect to all state variables. We know that equilibrium outcomes are functions of the state space. Thus we expand the deterministic economy in all state variables. But since we allow for arbitrarily many agents, we might also have arbitrarily many state variables.

The novel idea lies in exploiting the symmetry of decision rules across agents. If two agents are identical in their objectives, they respond identically in the same economic situation. For example, starting out from a case where both agents live under the same economic condition, a marginal increase in agent one's wealth will impact the decision of agent two the same way that agent one's decision would have been impacted by the same change in agent two's wealth. Exploiting the symmetry, we solve for the decision rules of all agents as a function of 
the entire distribution of individual states. In a second step, we also incorporate differences in individuals' objectives.

The last step makes the transition from the deterministic to the stochastic economy. Since shocks are part of the state space, the previous expansion delivers equilibrium reactions to known, deterministic changes in shocks. For example, the previous expansion would compute the asset price reaction if next period's productivity was above its steady-state level. To move to the stochastic economy, we integrate over all possible realizations of the shocks and weight them by their probability. From this logic it follows immediately that we need a higher-order expansion. If we were to resort to a first-order approximation, integrating over the first-order approximation would not affect equilibrium behavior since a linear solution is certaintyequivalent. Higher-order expansions bring in the effects of uncertainty. A second-order approximation reflects the effect of the variance of shocks, a cubic approximation additionally takes the skewness into account, and so on.

Our solution method is asymptotically valid and converges to the true solution. By adding higher moments, we can eventually recover the true policy function. In practice, of course, convergence is not complete. However, we have a means of testing the accuracy of our solution. We plug our approximation into the equilibrium conditions to check its optimality. The solution method is applicable to a wide range of applications. It applies whenever equilibrium or optimality conditions for a competitive equilibrium or dynamic programming problems imply that the choice variables are smooth functions of state variables. The dynamic programming problem or competitive equilibrium can feature arbitrarily many state variables and is thus interesting for a large set of economic applications. The implementation of constraints invalidates the smoothness of choices. However, for our method to apply, there are two ways to fix it. Either, one can smooth out the constraint such that there is no kink, or one can implement an endogenous borrowing constraint. Our economy provides an example for the latter.

We demonstrate the results from our solution method. First, we confirm previous research in finding that the impact of heterogeneity has an effect on the steady-state level of capital. Since agents face idiosyncratic risk, they respond by building up a buffer stock of precautionary savings. With aggregate risk this channel is enforced. Due the utility specification featuring constant relative risk aversion, agents increase their capital holdings further due to uncertain returns to capital.

Furthermore, we show the risk factors for this incomplete markets economy. Therefore, we present the expansion of a stochastic discount factor in closed form up to a given order. The 
expansion consists of standard risk factors such as total factor productivity and, if stochastic, its variance but also risk due to incompleteness of markets such as the variance of idiosyncratic labor income risk. This last factor only appears because there is a missing market that lets agents insure against their idiosyncratic conditions.

Lastly, we compare our solution method to a standard technique which replaces the actual law of motion to a linearized version. We solve an asset pricing economy in closed form, using the linearized law of motion, and the solution method of this paper. We find superior performance of our technique.

This paper contributes to a growing literature on introducing heterogeneity into economic models. Therefore, we relate to several strands of research. After the seminal works of ? and ?, the literature has focused on idiosyncratic risk with aggregate shocks. First, in special cases one might be able to find closed-form solutions as in ? and ?. Another promising idea is to use a multiplier approach to characterize features of the distribution of state variables across the population as in ? and ?. Other papers make simplifying assumptions on the number of agents and the number of possible shocks, as in ?. Special cases with closed-form solution can be used as a starting point for the expansion.

Most of the literature, however, is concerned with approximations. One idea is to replace the distribution of wealth by aggregate wealth only when calculating the equation of motion for aggregate variables. This method was developed in ? and inspired methods in the subsequent literature, for example in ?, ?, and ? where aggregate states and prices might influence the equation of motion. Alternatively, one might work with a limited history of shocks as in ?. Since we are particularly interested in the effect of distributions on equity prices and the effect on new financial securities, this approximation method is not appropriate for this research project.

Recently, alternative solution method for models with heterogeneous agents have been developed in ?, ?, ?, and ?. ? and ? parameterize the distribution of state variables. ? approximate the equilibrium on a lower-dimensional space. This paper develops a technique that does not require the specification of a class of distributions. Compared to ? and ?, the method in this paper has the advantages that we can study as many agents as desired whereas the number of agents is limited in their method. Furthermore, our method applies to models with many state variables and choices for each individual. Furthermore, the usual differences between perturbation and projection apply: our method returns quasi-analytical expressions, allows to prove theorems, get intuition for the impact of parameter changes, and is fast and simple. 
Our method builds on perturbation methods. These methods have been used in ?, ?, ?, ?, ?, ?, ?. Most recently, ? uses perturbation methods to study heterogeneity induced by private information. This solution method was also applied in ?. This paper, however, is not the first paper that attempts to use perturbation methods to analyze general equilibrium models with substantial heterogeneity. An alternative idea to the one in this paper has been explored by ? and ?. This work starts by restricting the state space from the outset. Instead, this paper is the first to recognize the symmetry of the problem and build a solution method that exploits it. No limitations on the state spae are required.

We also relate to the literature on the existence of equilibria. ? establish generic existence of equilibria by constructing a correspondence for expectations. In our economy, the rank of the span of assets cannot drop and, as a result, we obtain existence. ? and ? show conditions under which equilibria might fail to exist. ? establishes the existence of sequential equilibria for an economy with a continuum of agents with incomplete markets, aggregate risk, and hard borrowing constraints. ? proved existence of a recursive equilibrium for such an economy. Instead, we focus on endogenous borrowing constraints and smooth solutions and provide a recipe for proving existence in a broad class of economies. The reason why we can focus on

\section{Example: An economy with heterogeneous agents}

This section introduces our leading example, a standard dynamic stochastic general equilibrium model with idiosyncratic and aggregate risk.

\subsection{Households}

A finite number of $I$ households lives for an infinite number of periods indexed by $t$. Households are each endowed with one unit of time which they devote towards labor inelastically. While they are identical in their preferences, households differ in their productivity. Each period, a household receives an idiosyncratic shock to their productivity and thus their labor income. There is no asset available that lets households insure against their individual productivity. Therefore, agents can only partially insure against this shock by holding saving to buffer the shocks. A tradable contract consists of claims to capital which is risky due to aggregate productivity shocks. A second tradable contract is a bond with a risk-free payoff. Each household builds rational expectations and chooses streams of consumption, labor sup- 
ply, and capital holdings to maximize expected discounted utility

$$
\max _{c_{t}^{i}, k_{t+1}^{i}, b_{t+1}^{i}} \sum_{t=0}^{\infty} \beta^{t} E_{0}\left[u_{c}\left(c_{t}^{i}\right)-u_{k}\left(k_{t}^{i}, b_{t}^{i}\right)\right] \quad i=1, \ldots, I
$$

where $\beta$ is the time discount factor, $c_{t}^{i}$ is household $i$ 's consumption choice in period $t$, $k_{t}^{i}$ are household $i$ 's capital and $b_{t}^{i}$ bond holdings. The utility function is comprised of two additively separable parts. The first part is a standard utility function defined over individual consumption. To pin down the functional form, we impose constant relative risk aversion over consumption.

The second term in the utility specification incorporates a penalty function which serves two purposes. First, it incorporates an endogenous borrowing constraint. Second, it pins down the distribution of capital and bond holdings in the deterministic steady-state. ${ }^{1}$ We choose penalty functions for savings greater than some lower bound of the form

$$
u_{k}\left(k_{t}^{i}, b_{t}^{i}\right)=\nu_{1} \frac{1}{\left(k_{t}^{i}+b_{t}^{i}-\underline{k}\right)^{2}}+\nu_{2}\left(k_{t}^{i}-\bar{k}\right)^{2}+\nu_{3}\left(b_{t}^{i}\right)^{2}+\nu_{4}\left(k_{t}^{i}+b_{t}^{i}\right)
$$

where $\bar{k}$ denotes the target level of capital, $\underline{k}$ a lower bound on savings, and $\nu$. $>0$ parameters for the penalty function. We impose the ristriction $\nu_{4}=\nu_{1} \frac{2}{(\bar{k}-\underline{k})^{3}}$ to ensure that the penalty function has its global minimum at $\bar{k}$. Its derivative with respect to capital vanishes at its target level $\bar{k}$. For savings $k_{t}^{i}+b_{t}^{i}<\underline{k}$, we set the penalty to infinity.

The first part of the penalty function imposes the asymmetry between borrowing and saving. Borrowing is penalized in order to build in an endogenous borrowing constraint. ${ }^{2}$ Note that the penalty function only takes effect when going to higher expansions. A singularity appears in the penalty function while any Taylor series approximation will not have this feature. The singularity ensures though that higher-order approximations deliver stronger penalties.

The remaining three terms represent technical matters that should have negligible effects on choices. We will see later that the coefficients on these terms can be arbitrarily small. The second term is responsible for fixing the steady-state distribution of capital while the third part determines portfolio choice at the steady-state. The last term merely ensures that the choice between capital and bonds is well defined in the deterministic steady-state.

\footnotetext{
${ }^{1}$ The same goal can be achieved by endogenizing the interest rate or discount factor.

${ }^{2}$ An alternative approach would be to fix the budget constraint through an exogenous borrowing constraint which would result in a Lagrangian that takes a similar form. We do not pursue this approach because hard constraints can induce non-differentiability at certain points. A hard constraint can, however, be reformulated as a limiting sequence of penalty functions (see appendix ??).
} 
Households maximize utility subject to their budget constraint

$$
c_{t}^{i}+k_{t+1}^{i}+p_{t}^{b} b_{t+1}^{i}=\left(1+r_{t}^{k}\right) k_{t}+b_{t}+w_{t} \frac{e^{\psi_{t}^{i}}}{\Psi_{t}}
$$

The rate of return on capital is denoted by $r_{t}^{k}$, the price of bonds by $p_{t}^{b}$, and wages by $w_{t}$. To keep a concise notation, we introduce capital case letters for aggregate quantities of consumption, capital, bonds, and labor productivity

$$
C_{t}=\sum_{i=1}^{I} c_{t}^{i} \quad K_{t}=\sum_{i=1}^{I} k_{t}^{i} \quad B_{t}=\sum_{i=1}^{I} b_{t}^{i} \quad \Psi_{t}=\sum_{i=1}^{I} e^{\psi_{t}^{i}} .
$$

There is a shock to individual productivity denoted by $\psi_{t}^{i}$ which is independent and identically distributed across households. It follows a stochastic process of the form

$$
\psi_{t+1}^{i}=\bar{\phi}_{\psi}+\phi_{\psi} \psi_{t}^{i}+\phi_{\theta}\left(\psi_{t}^{i}\right) \sigma \theta_{t+1}^{i}
$$

The parameter $\phi_{\psi}$ governs the degree of persistence in the evolution of the shock. $\bar{\phi}_{\psi}$ adjusts the long-run mean and $\phi_{\theta}\left(\psi_{t}^{i}\right)$ governs the standard deviation of the shock which we allow to be a function of $\psi_{t}^{i}$. $\theta$ is white noise with unit variance. Agents cannot directly insure against this shock since there is no tradable asset contingent on individual labor productivity. Since we work with a finite number of households, the law of large numbers does not kick in and shocks might not be purely idiosyncratic. To circumvent this problem, we normalize productivity by average individual productivity $\Psi_{t}$ which ensures that markets clear.

\subsection{Technology}

Aggregate capital and labor enter the production process for the single consumption good which is characterized by a Cobb-Douglas production function. The parameter for the production function $\alpha$ leads to a functional form for output given by $Y=f(K, L, z)=e^{z} K^{\alpha} L^{1-\alpha}$ where $e^{z}$ denotes the shock to total factor productivity, $K$ aggregate capital and $L$ aggregate labor demand. The logarithm of total factor productivity follows an AR(1) process

$$
z_{t+1}=\bar{\phi}_{z}+\phi_{z} z_{t}+\phi_{\varepsilon} \sigma \varepsilon_{t}
$$

where the parameter $\phi_{z}$ determines the degree of mean reversion in total factor productivity. Firms maximize output net of costs for capital and labor. Given the constant returns to scale 
of the production function, wages and dividends pay their marginal product

$$
\begin{aligned}
& r_{t}^{k}=\alpha e^{z_{t}} K_{t}^{\alpha-1} L_{t}^{1-\alpha} \\
& w_{t}=(1-\alpha) e^{z_{t}} K_{t}^{\alpha} L_{t}^{-\alpha}
\end{aligned}
$$

Due to the shocks to total factor productivity, the returns to capital and wages are risky. Only one shock is driving the uncertainty of proceeds for both factor inputs. As a result, labor income and returns to capital are conditionally positively correlated.

We specify all shocks to $z$ and $\psi$ to have a uniform distribution on a compact support which means that there are bounds $\underline{\varepsilon}$ and $\bar{\varepsilon}$ resp. $\underline{\theta}$ and $\bar{\theta}$. The support increases with the scaling of the standard deviations $\sigma$. We use $\bar{\phi}_{z}$ and $\bar{\phi}_{\psi}$ to adjust the mean of the random variables $e^{z}$ and $e^{\psi}$ by setting $\bar{\phi}_{z}=\log \left((\bar{\varepsilon}-\underline{\varepsilon}) /\left(e^{\bar{\varepsilon}}-e^{\underline{\varepsilon}}\right)\right)$, analogously for $\psi$.

\subsection{Definition of equilibrium}

The aggregate resource constraint is given by the following equation

$$
C_{t}+K_{t+1}-(1-\delta) K_{t}=Y_{t}
$$

which shows how current output and depreciated capital can be used for consumption or next period's capital stock. The derivation follows from the households' budget constraints and the two market clearing conditions for capital

$$
\sum_{i=1}^{I} k_{t}^{i}=K_{t}
$$

and bonds being in zero net supply

$$
\sum_{i=1}^{I} b_{t}^{i}=0 .
$$

Optimal choices obey the first-order conditions

$$
u_{c}^{\prime}\left(c_{t}^{i}\right)=\beta E_{t}\left[\left(1+r_{t+1}^{k}\right) u_{c}^{\prime}\left(c_{t+1}^{i}\right)-u_{k}^{(1)}\left(k_{t+1}^{i}, b_{t+1}^{i}\right)\right]
$$

and

$$
u_{c}^{\prime}\left(c_{t}^{i}\right)=\beta E_{t}\left[\left(1+r_{t}^{b}\right) u_{c}^{\prime}\left(c_{t+1}^{i}\right)-u_{k}^{(2)}\left(k_{t+1}^{i}, b_{t+1}^{i}\right)\right]
$$

where $u_{k}^{(l)}$ denotes the derivative with respect to the $l$-th argument and $r_{t}^{b}=1 / p_{t}^{b}-1$ the 
return on bonds. If we set all parameters $\nu$. to zero in the utility function, these optimality conditions result in standard Euler equations. With the parameters $\nu$ being non-zero, we impose an endogenous borrowing constraint. Since the penalty function, which imposes the borrowing constraint in the following period, depends on next period's capital stock, a marginal unit of consumption today marginally increases the expected penalty.

The state space of this economy consists of the set of individidual capital holdings of each of the $I$ households, their bond holdings, and the level of their individual productivity. Furthermore, we keep track of aggregate productivity. To define the state space in a concise manner, we introduce the following notational convention. We denote vectors by a small case bold letter and a matrix by an upper case bold font letter. Furthermore, we clarify whether the state space belongs to a stochastic or deterministic economy by making each function dependent on $\sigma \in[0,1]$, a variable that scales the standard deviation of all shocks proportionately. When the standard deviation of shocks $\sigma$ equals one, we refer to the stochastic economy. The deterministic counterpart is denoted by $\sigma=0$. An element of the state space is denoted by $\left(\mathbf{X}_{t}, \mathbf{z}_{t}\right) \in \mathbb{R}^{3 I+1}$. An equilibrium consists of price, choice, and transition functions. We denote transition functions by $\mathbf{X}\left(\mathbf{X}_{t}, \mathbf{z}_{t}, \sigma\right)$ and $\mathbf{z}\left(\mathbf{X}_{t}, \mathbf{z}_{t}, \sigma\right)$ or, in a slight abuse of notation, by $\mathbf{X}(\sigma)$ resp. $\mathbf{z}(\sigma)$. And to simplify the notation further, we denote an element $(\mathbf{X}(\sigma), \mathbf{z}(\sigma), \mathbf{C}(\sigma), \mathbf{p}(\sigma))$ by $\mathbf{B}(\sigma)$.

We collect all equilibrium conditions in a single operator $G$

$$
G(\mathbf{B}(\sigma), \sigma)\left(\mathbf{X}_{t}, \mathbf{z}_{t}\right)=\left(\begin{array}{l}
E_{t}\left[g_{1}\left(\mathbf{X}_{t}, \mathbf{z}_{t}, \mathbf{X}(\sigma), \mathbf{z}(\sigma), \mathbf{C}(\sigma), \mathbf{p}(\sigma)\right)\right] \\
\mathbf{X}\left(\mathbf{X}_{t}, \mathbf{z}_{t}, \sigma\right)-g_{2}\left(\mathbf{X}_{t}, \mathbf{z}_{t}, \mathbf{C}, \mathbf{p}\right)
\end{array}\right) .
$$

The operator takes the state variables as its inputs along with the collection of choice variables $\mathbf{C}$, price functions $\mathbf{p}$, and transitions for state variables and shocks as functions of the state space. The operator $g^{1}$ consists of all $2 \times I$ Euler equations stacked in one large vector along with the budget constraints. $g^{2}$ stacks the equation of motion for aggregate capital (??), its definition, market clearing for bonds, the stochastic processes for $z$ and $\psi$, and the conditions for wages and returns paying their marginal products.

\section{Definition 1 (Definition of Equilibrium for Economy)}

An equilibrium of the economy is a collection of consumption functions and portfolio choice decision $\mathbf{C}$ and price functions $\mathbf{p}$ together with the law of motion such that $G(\mathbf{B}(\sigma), \sigma)\left(\mathbf{X}_{t}, \mathbf{z}_{t}\right)=$ 0 .

If we find such a function, all agents are at their optimum. There is one point in the state space which is of particular interest. 


\section{Definition 2 (Deterministic Steady-State)}

$A$ deterministic steady-state is a point in the state space $\left(\mathbf{X}_{0}, \mathbf{z}_{0}, 0\right)$ such that each household's first-order conditions are satisfied, consumption is constant, and capital does not change.

\section{Existence}

This section discusses existence and uniqueness of equilibria for the economy of the previous sections. Since the method for proving these results is more general than the particular application, we lay out the mathematical foundations that should make the methodology for proofs as portable as possible. We demonstrate existence and uniqueness results in two parts. First, we deal with existence locally around the deterministic case. Second, we deal with the case of large risk.

\subsection{Local existence}

We exploit the differentiability of equilibrium conditions to show existence of equilibria for our economy with small risks. We determine optimal policy functions for the stochastic case using the implicit function theorem around the deterministic case. The solution consists of functions of the state space. Hence we need to build derivatives with respect to a function. We review the necessary concepts in this section.

The starting point for our analysis is the solution to the deterministic economy, i.e. $\sigma=0$. The existence of the solution is known for this case and we obtain differentiability of optimal policies for this case. For example, the analysis in ? shows that the optimal policy will be infinitely often differentiable, i.e. it lies in the space $\mathcal{C}^{\infty}$. Furthermore, by applying the contraction mapping theorem for this case, the optimal policy is unique. We stick to the class of continuously differentiable functions and thus rule out $L_{1}, L_{2}$, and $L_{\infty}$ spaces.

Our economy has an infinitely often differentiable solution to the deterministic economy. Once we introduce risk, we have to make sure that there are no unit roots and nobody can run a Ponzi scheme. These conditions are insured by the endogenous borrowing constraint in (??). Furthermore, our economy does not run the risk that the span of the assets can ever collapse since prices are constant and dividends risky.

The operator $G$ in equation (??) defines the equilibrium operates on a Banach space (i.e. a complete normed vector space) if we define the choice, transition, and price functions on an appropriately chosen compact domain $\Omega$. We can thus write $G: \mathcal{B}_{1} \times \mathbb{R} \rightarrow \mathcal{B}_{2}$ where 
$\mathcal{B}_{1}=\mathcal{C}^{k}[\Omega]^{6 I+3}$ and $\mathcal{B}_{2}$ are Banach spaces. The Banach space $\mathcal{B}_{1}$ consists of elements $\mathbf{B}(\sigma)$ defined above equation (??). The domain $\Omega$ is a compact subset of $\mathbb{R}^{3 I+1}$ (the exact choice is presented in appendix ??). We define $\Omega_{\sigma}=\Omega \times[-1,1]$ which is a combination of elements in the state space and scaling of standard deviations $\omega_{\sigma}=(\omega, \sigma)$. The Banach space possesses the norm

$$
\|B(\sigma)\|_{\mathcal{B}_{1}}=\sum_{\mathbf{U} \in\{\mathbf{X}, \mathbf{z}, \mathbf{C}, \mathbf{p}\}} \sum_{\kappa=0}^{k}\left(\sup _{\omega_{\sigma} \in \Omega_{\sigma}}\left|\mathbf{U}^{\kappa}\left(\omega_{\sigma}\right)\right|\right)
$$

where the superscript $\kappa$ indicates the order of the derivative. To investigate the behavior close to a solution, we define derivatives of the operator $G$. Since the operator is defined over functions, we define the Fréchet derivative of the operator.

\section{Definition 3 (Fréchet derivative)}

$A$ bounded linear map $D G\left(\mathbf{B}^{*}, \sigma^{*}\right): \mathcal{B}_{1} \times \mathbb{R} \rightarrow \mathcal{B}_{2}$ is called Fréchet derivative of $G$ at $\left(\mathbf{B}^{*}, \sigma^{*}\right)$ if

$$
G\left(\mathbf{B}^{*}+\boldsymbol{\epsilon}, \sigma^{*}\right)=G\left(\mathbf{B}^{*}, \sigma^{*}\right)+D G\left(\mathbf{B}^{*}, \sigma^{*}\right) \boldsymbol{\epsilon}+o\left(\|\boldsymbol{\epsilon}\|_{\mathcal{B}_{1}}\right)
$$

where $o\left(\|\boldsymbol{\epsilon}\|_{\mathcal{B}_{1}}\right)$ means $\frac{o\left(\|\boldsymbol{\epsilon}\|_{\mathcal{B}_{1}}\right)}{\|\boldsymbol{\|}\|_{\mathcal{B}_{1}}} \rightarrow 0$ for $\|\boldsymbol{\epsilon}\|_{\mathcal{B}_{1}} \rightarrow 0$.

We define higher-order derivatives, as long as they exist, by induction.

\section{Definition 4 (Higher-order derivatives)}

$A$ higher-order derivative is a bounded linear map $D^{m} G\left(\mathbf{B}^{*}, \sigma^{*}\right): \mathcal{B}_{1} \times \mathbb{R} \rightarrow \mathcal{B}_{2}$ defined as $D^{m} G=D\left(D^{m-1} G\right)$.

Equipped with derivatives, we can now make use of the fact that the Implicit Function Theorem carries over to Banach spaces. The idea will be that we parameterize our economy with the standard deviation of shocks such that a value of zero represents the deterministic economy and a value of one the stochastic economy. For the local results, we simply focus on a ball around the deterministic economy. We denote the Fréchet derivative of $G$ with respect to the element in the Banach space $\mathcal{B}_{1}$ by $D_{1} G$. Then the following theorem holds.

\section{Theorem 1 (Implicit Function Theorem for Banach Spaces)}

Suppose $G: \mathcal{B}_{1} \times \mathbb{R} \rightarrow \mathcal{B}_{2}$ is continuously differentiable in a neighborhood of the point $\left(\mathbf{B}^{*}, 0\right)$ and that $G\left(\mathbf{B}^{*}, 0\right)=0$. Further suppose that the map $D G$ is a linear homeomorphism of $\mathcal{B}_{1}$ onto $\mathcal{B}_{2}$. Then there exist open subsets around $\mathbf{B}^{*}$ and 0 such that $G(\mathbf{B}(\sigma), \sigma)=0$.

The assumption of the Fréchet derivative being a linear homeomorphism simply states that the derivative and its inverse at the point of expansion exist and constitute linear mappings 
between the two Banach spaces. The proof of the theorem is an application of the Contraction Mapping Theorem and can be found in standard nonlinear analysis textbooks, for example $?$.

\section{Proposition 1 (Existence of local solutions)}

The equilibrium of the incomplete markets economy of section ?? exists for small amounts of risk. Furthermore, the consumption and savings functions are infinitely often differentiable, i.e. elements of $\mathcal{C}^{\infty}$.

The proof is essentially given by the Implicit Function theorem. Further details are discussed in appendix ??. As corollary to our proposition, we find that the solution is unique.

Corollary 1 (Uniqueness) The solution of the economy with small risk is unique.

\subsection{Global existence}

We now establish existence of equilibria for an economy with large risks. As in the previous section, the scaling variable for the standard deviations $\sigma$ delivers a mapping between the deterministic and the stochastic economy. We show that the solution can be extended beyond the domain of the Implicit Function Theorem. Extending from a simpler case to the desired solution is generally referred to as a homotopy method and these ideas have been used to prove existence of partial differential equations. We adapt the theory to make it available for our purposes.

We define a bounded open subset $U \subset \mathcal{B}_{1} \times[0,1]$ and let $U(\sigma):\left\{u \in \mathcal{B}_{1} \mid(u, \sigma) \in U\right\}$ be the subset of $\mathcal{B}_{1}$ corresponding to a particular $\sigma$. We study the map $G: \mathcal{B}_{1} \times[0,1] \rightarrow \mathcal{B}_{2}$ introduced in equation (??) to establish solutions to the equation $G(\mathbf{B}, \sigma) \equiv 0$.

To prove existence, we apply the Leray-Schauder continuation theorem for which we follow the discussion in ?. Therefore, we define the operator $\hat{G}(\mathbf{B}, \sigma)=G(\mathbf{B}, \sigma)+\mathbf{B}$ such that the equation to solve becomes $G(\mathbf{B}, \sigma)=\hat{G}(\mathbf{B}, \sigma)-\mathbf{B}=0$. If we show that the operator $\hat{G}$ is completely continuous on the closure of $U, \bar{U}$, and furthermore $G(\mathbf{B}, \sigma) \neq 0$ for all $\sigma \in[0,1]$ and $\mathbf{B}$ on the boundary of $U(\sigma), \partial U(\sigma)$, then we can trace the solution all the way from the deterministic $(\sigma=0)$ to the stochastic $(\sigma=1)$ economy. For a more detailed discussion of the underlying theory, see ?.

Theorem 2 (Leray-Schauder Continuation)

If $\hat{G}$ is completely continuous and $G(\mathbf{B}, \sigma) \neq 0$ for all $\sigma \in[0,1]$ and all $\mathbf{B}$ on the boundary 
of $U(\sigma)$, then there exists a continuum $C \subseteq\{(\mathbf{B}, \sigma) \in \bar{U} \mid G(\mathbf{B}, \sigma)=0\}$ such that $C \cap U(0) \neq$ $\emptyset \neq C \cap U(1)$.

Once we apply this theorem to our economy, we get the existence for the economy with large risks. The challenge here is to define the subsets in such a way that we are able to show the assumptions necessary to apply the Leray-Schauder continuation theorem.

\section{Proposition 2 (Existence of Equilibria with Large Risks)}

The equilibrium of our incomplete markets economy with standard deviations $\sigma_{z}<\bar{\sigma}_{z}$ and constant standard deviation $\sigma_{\psi}<\bar{\sigma}_{z}$ also exists for large risks $\sigma=1$.

A proof can be found in appendix ??. Essentially, the proof amounts to defining the operator on the right subset $U$ on which we can show complete continuity. The subset needs to be designed in a way that keeps all the solutions within the set.

\section{Numerical method}

This section uses the previous analysis to develop a solution method for incomplete market models with substantial heterogeneity. We apply perturbation methods to derive a higherorder approximation to the solution. As in the previous section, we first shut down all uncertainty and find the deterministic steady-state of the economy. Then we exploit the symmetry of the model to keep the high-dimensionality of the model tractable.

\subsection{General Setup}

This paper's solution method handles competitive equilibria as well as dynamic programming problems. To demonstrate the generality of the solution method, we define a matrix of individual state variables $\mathbf{X}_{t} \in \mathbb{R}^{C \times I}$ and a vector of aggregate shocks $\mathbf{z}_{t} \in \mathbb{R}^{Z}$ where $C$ denotes the number of individual state variables and $Z$ the number of aggregate shocks. We write the first-order conditions (resp. Bellman equation) along with the equation of motions, market clearing conditions, and budget constraints in the general form given in (??). To apply our method, we require three main assumptions for the models.

First, we require the model to feature smooth policy functions. We apply perturbation methods to the problem which build a Taylor series expansion of the optimal policies around a deterministic steady-state. In many economic problems, optimal policies are analytic which implies they possess all derivatives. If policies are smooth, the Taylor series converges within 
a radius and we obtain a global approximation within that region. Second, we require the economy to have a well-defined ergodic distribution to avoid unit roots. And third, we require the existence of a deterministic steady-state at which all agents are identical. ${ }^{3}$

For most purposes, we impose complete symmetry on the functional $g^{1}$. For example, in our competitive equilibrium all agents' first-order conditions are the same. If we denote $\mathbf{X}^{i \leftrightarrow j}$ the matrix of state variables where we exchange the state variables of agent $i$ with agent $j$ and vice versa and the same for policy functions $P^{i \leftrightarrow j}$, then we can express the symmetry requirement as

$$
g_{k}^{i}\left(\mathbf{X}_{t}, \mathbf{z}_{t}, \mathbf{X}(\sigma), \mathbf{z}(\sigma), \mathbf{C}(\sigma), \mathbf{p}(\sigma)\right)=g_{k}^{j}\left(\mathbf{X}_{t}^{i \leftrightarrow j}, \mathbf{z}_{t}, \mathbf{X}(\sigma)^{i \leftrightarrow j}, \mathbf{z}(\sigma), \mathbf{C}(\sigma)^{i \leftrightarrow j}, \mathbf{p}(\sigma)\right) \quad k=1,2 .
$$

As a consequence, we can restrict ourselves to building an approximate solution for only one agent.

\subsection{Deterministic steady-state}

To solve for the deterministic steady-state, we set the standard deviation of all shocks to zero. Since agents are heterogeneous only with respect to their idiosyncratic labor income shocks in our model, the deterministic steady-state features identical agents and no heterogeneity. At this point, the penalty function approach we used for our example comes in. Without any penalty, the steady-state distribution of capital would be indeterminate. To see this, we look at the steady-state condition

$$
1-\delta+f_{K}\left(K_{t}, L_{t}, 0\right)=\frac{1}{\beta}=1+r_{t}^{b}
$$

which only depends on aggregate capital and not on the distribution of wealth. In our setup, however, agents obtain small disutility from deviating from a target level of capital. This penalty function implies that there is a unique distribution of capital in the deterministic steady-state, irrespective of how small the penalty from deviating from the target is.

Due to the portfolio choice problem, a second penalty term is needed to avoid indeterminacies. arises. As equation (??) shows, the return to capital and bonds must be identical to rule out arbitrage opportunities. The perfect substitutability implies an indeterminacy of the portfolio in the deterministic steady-state. The introduction of a small penalty for deviating

\footnotetext{
${ }^{3}$ Imposing identical agents in that steady-state does not mean that we cannot allow for heterogeneity. We can expand in the dimension in which agents are heterogeneous, for example with respect to their risk aversion.
} 
from identical portfolios across agents circumvents this problem. ${ }^{4}$

\subsection{Higher-order expansion}

Computing a higher-order Taylor series for the equilibrium policy functions, quantities, and prices is essential to our solution method. There are two reasons for it. First, heterogeneity manifests its impact only in higher-order terms and second, so does stochasticity. To compute high-order derivatives, a high precision arithmetic might be necessary as ? points out.

A Taylor series expansion of high order serves as a good approximation to equilibrium outcomes. For analytic functions, the approximation will converge within the radius of convergence when we increase the order of the expansion. In practice, of course, we have to truncate the Taylor series at a finite level. But the stage at which we stop can be endogenous to the accuracy of the solution.

Taylor expansions are at the heart of perturbation methods and we state them using the standard multi-index notation. For a reference, see, for example, ? (p. 3-16). We denote a C-tuple of integers by $\iota_{i}=\left(\iota_{i 1}, \iota_{i 2}, \ldots, \iota_{i C}\right)$ to index individual states for agent $i$. Let $\mathbf{I}=\left\{\boldsymbol{\iota}_{1}, \ldots, \boldsymbol{\iota}_{I}\right\}$ be the collection of such indices for all agents. Furthermore, $\mathbf{j}$ is a Ztuple of integers to index all aggregate shocks. The order of differentiation is then given by $\|\mathbf{I}\|+|\mathbf{j}|+k$ where $\|\mathbf{I}\|=\sum_{i=1}^{I} \sum_{\chi=1}^{C} \iota_{i \chi}$ and $|\mathbf{j}|=\sum_{\xi=1}^{Z} \mathbf{j}_{\xi}$. We also define the product of all entries $\mathbf{I} !=\prod_{i=1}^{I} \prod_{\chi=1}^{C} \iota_{i \chi}$ and $\mathbf{j} !=\prod_{\xi=1}^{Z} \mathbf{j} \xi$. A concise notation for a derivative of choice $\mathbf{C}$ reads

$$
\mathbf{C}^{(\mathbf{I}, \mathbf{j}, k)}\left(\mathbf{X}^{0}, \mathbf{z}^{0}, 0\right)=\left(\prod_{i=1}^{I} \prod_{\chi=1}^{C} \partial_{\iota_{i \chi}}\right)\left(\prod_{\xi=1}^{Z} \partial_{\xi}\right) \partial_{k} \mathbf{C}\left(\mathbf{X}^{0}, \mathbf{z}^{0}, 0\right)
$$

where $\partial_{\boldsymbol{\iota}_{i \chi}}=\partial^{\boldsymbol{\iota}_{i \chi}} / \partial \mathbf{X}_{i \chi}^{\boldsymbol{\iota}_{i \chi}}, \partial_{\xi}=\partial^{j_{\xi}} / \partial \mathbf{z}_{\xi}^{j_{\xi}}$, and $\partial_{k}=\partial^{k} / \partial \sigma^{k}$. Lastly, we define the monomials in the Taylor series accordingly. Let $\left(\mathbf{X}-\mathbf{X}^{0}\right)^{\mathbf{I}}=\prod_{i=1}^{I} \prod_{\chi=1}^{C}\left(\mathbf{X}_{i, \chi}-\mathbf{X}_{i \chi}^{0}\right)^{\iota_{i \chi}}$ and analogously $\left(\mathbf{z}-\mathbf{z}^{0}\right)^{\mathbf{j}}=\prod_{\xi=1}^{Z}\left(\mathbf{z}_{\xi}-\mathbf{z}_{\xi}^{0}\right)^{\mathbf{j}_{\xi}}$.

Once we know the derivatives at a specific point, we can recover the choice variable of the $\varpi$ 's choice of agent $i$ from the Taylor series

$$
\mathbf{C}_{\varpi}^{i}(\mathbf{X}, \mathbf{z}, \sigma)=\sum_{o=0}^{\infty} \sum_{\|\mathbf{I}\|+|\mathbf{j}|+k=o} \frac{1}{\mathbf{I} ! \cdot \mathbf{j} ! \cdot k !} \mathbf{C}^{(\mathbf{I}, \mathbf{j}, k)}\left(\mathbf{X}^{0}, \mathbf{z}^{0}, 0\right)\left(\mathbf{X}-\mathbf{X}^{0}\right)^{\mathbf{I}}\left(\mathbf{z}-\mathbf{z}^{0}\right)^{\mathbf{j}} \sigma^{k} .
$$

\footnotetext{
${ }^{4} \mathrm{~A}$ penalty term might not be necessary for a stochastic economy since the risk premium guarantees a unique portfolio allocation. For the existence, however, we would have to work with the Bifurcation Theorem instead of the Implicit Function Theorem.
} 
Note that equation (??) displays a function over the state space.

To obtain derivatives at the deterministic steady-state, we employ perturbation methods. Ultimately, we are interested in a solution to equation (??). Perturbation methods tell us to take derivatives of each equation with respect to all state variables successively and evaluating the resulting equations at the deterministic steady-state. By the chain rule, we obtain equations for the derivatives of the policy function at the deterministic steady-state which we can then solve for. Plugging them into equation (??) results in an approximation of the policy function. To give an example, take a first-order condition of the form (??). We take the derivative with respect to the first individual state variable $x_{1}^{1}$

$$
u_{c}^{\prime \prime} \frac{\partial c_{t}^{i}}{\partial x_{1}^{1}}-\beta \frac{\partial r_{t}^{b}}{\partial x_{1}^{1}} u_{c}^{\prime}+\left(1+r_{t}^{b}\right) u_{c}^{\prime \prime} \frac{d c_{t+1}^{i}}{d x_{1}^{1}}-u_{k}^{12} \frac{\partial k_{t+1}^{i}}{\partial x_{1}^{1}}-u_{k}^{22} \frac{\partial b_{t+1}^{i}}{\partial x_{1}^{1}}=0
$$

where arguments of the utility function are suppressed. The derivative $d c_{t+1}^{i} / d x_{1}^{1}$ is a long object since $c_{t+1}^{i}$ is a function of $\mathbf{X}_{t+1}$ and $\mathbf{z}_{t+1}$ for which we have to apply the chain rule.

More generally, we need to take derivatives of every equilibrium equation $g_{i}^{1}$ or $g_{i}^{2}$

$$
\frac{d g_{i}}{d x_{1}^{1}}=\frac{\partial g_{i}}{\partial x_{1}^{1}}+\frac{\partial g_{i}}{\partial \mathbf{C}_{t}} \frac{\partial \mathbf{C}_{t}}{\partial x_{1}^{1}}+\frac{\partial g_{i}}{\partial \mathbf{p}_{t}} \frac{\partial \mathbf{p}_{t}}{\partial x_{1}^{1}}+\frac{\partial g_{i}}{\partial \mathbf{X}_{t+1}} \frac{\partial \mathbf{X}_{t+1}}{\partial x_{1}^{1}}+\frac{\partial g_{i}}{\partial \mathbf{z}_{t+1}} \frac{\partial \mathbf{z}_{t+1}}{\partial x_{1}^{1}}+\frac{\partial g_{i}}{\partial \mathbf{C}_{t+1}} \frac{d \mathbf{C}_{t+1}}{d x_{1}^{1}}+\frac{\partial g_{i}}{\partial \mathbf{p}_{t+1}} \frac{d \mathbf{p}_{t+1}}{d x_{1}^{1}}
$$

and plug in steady-state values.

Now we use the fact that all the partial derivatives of $g_{i}^{1}$ are known. They are simply the derivatives of the equilibrium conditions (in the example of the Euler equation, these derivatives entail differentiating marginal utilities). When evaluating them at the deterministic steady-state, the only remaining variables in the differentiated equilibrium conditions are the derivatives of the optimal policies $\mathbf{C}$ and prices $\mathbf{p}$ at the deterministic steady-state. These are the coefficients in the Taylor series of the optimal policies.

The key innovation of this paper lies in recognizing the symmetry of the problem. In principle, we would have to start with agent one, differentiate his first-order conditions with respect to each agent's state variables, move to agent two and so on. However, we do not have to go through this entire process. There are two crucial ways in which the problem is symmetric. First, all derivatives with respect to state variables of other agents than the one whose policy we approximate are identical. For example, when differentiating agent one's firstorder condition, there are only two different coefficients in the first-order expansion: The derivative with respect to the agent's own state varaibles and those of any other agent. These two numbers are sufficient because the coefficients on other agents' state variables have to 
be the same since they are all given the same fundamentals. Second, we only have to take all derivatives of first-order conditions for agent one. Agent two's first-order conditions look identical and thus lead to the same result. The symmetry here is that agent one's response to a marginal increase in agent two's state variable is the same as agent two's response to a marginal increase in agent one's state variable. This carries over to all derivatives.

Exploiting this symmetry, a first-order approximation requires two coefficients to be computed for each state variable. One coefficient returns the change in policy of an agent in response to a change in her own wealth. The second coefficient asks for this agent's reaction in response to a change in the state variable by somebody else. For the second order term, the system becomes slightly more complex. For each state variable, we have to compute four values: an agent's change in response to a change in her state variable to a change in response to her wealth, an agent's change in response to a change in her state variable to a change in response to somebody else's state variable, an agent's change in response to a change in somebody else's state variable to a change in response this person's state variable, and an agent's change in response to a change in somebody else's state variable to a change in response to a third person's state variable. Increasing the order, we have quadratic growth in the number of coefficients which stays manageable. Although the economic interpretation is tedious, solving the system of equations is straightforward. From the second order on, the system of equations for the unknown coefficients is linear.

A first-order approximation implements standard linearization which is not sufficient for our purposes. Due to linearity, heterogeneity does not affect equilibrium outcomes because under these rules, the average choice is the choice of the average person. Heterogeneity only enters through higher-order terms, starting with a second-order approximation. For the same reason, stochasticity impacts equilibrium only through higher-order terms. The first-order approximation is certainty equivalent while higher-order terms add the effects of variance, skewness, and higher moments.

\subsection{Uncertainty}

Having obtained a high order approximation of the deterministic economy, we move towards its stochastic counterpart. We accomplish the transition by varying the scaling variable for the standard deviation of shocks $\sigma$.

Taking a first-order expansion with respect to the scaling of the standard deviation produces coefficients which are all zero. The reason lies in the fact that the first-order expansion of the standard deviation introduces shocks only into the linearized, and thus certainty equivalent, 
economy. Hence, uncertainty does not play a role. Only through second- and higher-order terms do we recover the solution to the stochastic system. The second-order term introduces shocks into the quadratic economy. This approximation is no longer certainty equivalent and uncertainty takes effect. To be more precise, the second-order term introduces a constant effect due to the variance of shocks, the third-order term recovers the reaction to skewness and time-variation in the variance of shocks, and so on.

We can interpret the way uncertainty enters the equilibrium as effectively altering the coefficients in the Taylor series. Building the expansion with respect to the standard deviation of shocks effectively alters the coefficients to the Taylor series of the deterministic system. To see this, we truncate equation (??) after $O$ terms and rewrite it in the form

$$
\mathbf{C}_{\varpi}^{i}(\mathbf{X}, \mathbf{z}, \sigma)=\sum_{o=0}^{\infty} \sum_{\|\mathbf{I}\|+|\mathbf{j}|=o} \frac{1}{\mathbf{I} ! \cdot \mathbf{j} !}\left(\sum_{k=0}^{\infty} \frac{\mathbf{I} ! \cdot \mathbf{j} !}{\mathbf{I} ! \cdot \mathbf{j} ! \cdot k !} \mathbf{C}^{(\mathbf{I}, \mathbf{j}, k)}\left(\mathbf{X}^{0}, \mathbf{z}^{0}, 0\right) \sigma^{k}\right)\left(\mathbf{X}-\mathbf{X}^{0}\right)^{\mathbf{I}}\left(\mathbf{z}-\mathbf{z}^{0}\right)^{\mathbf{j}}
$$

The rearrangement demonstrates that the expansion of the stochastic system looks just like the deterministic system except that the coefficients (in brackets) contain a "correction term" for the stochasticity of the function.

We can see this term graphically as depicted in figure ??. In the second-order, the function shifts while the third-order term would also tilt the function while even higher orders change its curvature.

Figure 1: Perturbation methods build an approximation in state variables around the deterministic steady-state (thick solid line). The expansion with respect to the standard deviation shifts (second order) and tilts this line (third order). 


\subsection{The Law of Motion}

Perturbation methods deliver a polynomial representation of the approxmation. The law of motion is no exception to this rule. In our approximate solution, the equation of motion is not only a function of an aggregate statistic of state variables but the entire distribution as the theory would tell us. With every increase in the order of approximation, our solution method includes the corresponding moments from the distribution of state variables. In this sense, the solution method proposes a set of approximating statistics with which to approximate policy functions. As an additional feature, a better approximation adds moments to the previous approximation without the necessity to recompute previous approximations.

The first-order expansion results in a law of motion of the form

$$
\begin{gathered}
\frac{1}{I} K_{t+1} \approx k_{0}+\left(k_{\mathbf{X}_{11}}^{1}-k_{\mathbf{X}_{21}}^{1}+I k_{\mathbf{X}_{21}}^{i}\right)\left(\mathbf{X}_{1}-\overline{\mathbf{X}}_{11}\right)+\left(k_{\mathbf{X}_{12}}^{1}-k_{\mathbf{X}_{22}}^{1}+I k_{\mathbf{X}_{22}}^{i}\right)\left(\mathbf{X}_{2}-\overline{\mathbf{X}}_{12}\right) \\
+I k_{\mathbf{z}_{1}}^{1}\left(\mathbf{z}_{1}-\overline{\mathbf{z}}_{1}\right)+I k_{\mathbf{z}_{2}}^{1}\left(\mathbf{z}_{2}-\overline{\mathbf{z}}_{2}\right)+I k_{\mathbf{z}_{3}}^{1}\left(\mathbf{z}_{3}-\overline{\mathbf{z}}_{3}\right)+\ldots
\end{gathered}
$$

where $\mathbf{X}_{j}=\frac{1}{I} \sum_{i=1}^{I} \mathbf{X}_{i j}, \overline{\mathbf{X}}_{1}$. is the value of the state variable at the deterministic steadystate, and $k_{\mathbf{X}_{11}}^{1}=\frac{\partial k^{1}}{\partial \mathbf{X}_{11}}$, see appendix ?? for a derivation.

The second-order approximation depends both on the cross-sectional variance of individual state variables as well as a quadratic term in aggregate state variable. It thus depends not only on the cross-section but also nonlinearly on the time-series variation of aggregate quantities.

The above expression is not particular to the law of motion. Any function which depends identically on agents' choices, will be approximated in this fashion. Appendix ?? contains details.

\subsection{Distribution of Equilibrium Variables}

Given our approximation method, we compute the distribution of any equilibrium outcomes or nonlinear functions thereof. We therefore combine perturbation methods with a non-linear change of variables. ${ }^{5}$ For example, from capital and bond holdings we compute portfolio weights or Sharpe ratios of individual portfolios.

Suppose we have some economic variable of interest which is a nonlinear function $h(\mathbf{X}, \mathbf{z}, \mathbf{P})$ of the state variables and choices which we approximate with a Taylor series. The coefficients

\footnotetext{
${ }^{5}$ ?, ?, and ? explain nonlinear changes of variables in conjunction with perturbation methods.
} 
can be computed as follows

$$
\frac{d h}{d x_{s}^{i}}=\frac{\partial h}{\partial \mathbf{X}} \frac{\partial \mathbf{X}}{\partial x_{t}^{i}}+\frac{\partial \mathbf{C}}{\partial \mathbf{X}} \frac{\partial \mathbf{X}}{\partial x_{t}^{i}}+\frac{\partial \mathbf{p}}{\partial \mathbf{X}} \frac{\partial \mathbf{X}}{\partial x_{t}^{i}}
$$

and analogously for other state variables. All partial derivatives of $h$ are given through its functional form while the derivatives of state variables, choices, and prices were previously computed through perturbation methods.

The computation of the coefficient is trivial once we make the observation that the first term is given by the derivative of $H$ (which is given) and the second one has already been computed in the previous approximation. Thus, computing the distribution of any variable of interest within the economy is not more intricate than computing the distribution of capital.

\subsection{Accuracy}

The solution method comes with a natural way to check for its accuracy. The equilibrium conditions are satisfied when the functional $G$ in equation (??) returns zero values for all of its components. Since we have asymptotic validity of the solution method, we specify a tolerance as a threshold for the error. Once the error is below the tolerance in some norm, we terminate the approximation process.

To get a meaningful measurement for the error, it makes sense to normalize the optimality conditions such that they are unit-free. For example, we rewrite the Euler equation (??) in the form

$$
\beta E_{t}\left[\left(1+r_{t+1}^{k}\right) \frac{u_{c}^{\prime}\left(c_{t+1}^{i}\right)}{u_{c}^{\prime}\left(c_{t}^{i}\right)}-\frac{u_{k}^{(1)}\left(k_{t+1}^{i}, b_{t+1}^{i}\right)}{u_{c}^{\prime}\left(c_{t}^{i}\right)}\right]=0
$$

to avoid the error scaling with marginal utility. This measurement provides a way to check for accuracy after adding an order of approximation. Thus one can decide at each step whether the approximation suffices the criteria or not. As an additional benefit, there is no need to recompute previous orders after each step. The approximation method keeps previous coefficients unaltered when refining the solution.

\section{Results}

This section summarizes the findings for the particular economy of section ??. We first discuss the choice of functional forms and parameter values. Then we show the accuracy of the solution method before discussing findings with respect to the two versions of the 
economy.

\subsection{Calibration}

Most of the parameters and functional forms are standard in the literature. In large parts, there is a concensus on how to calibrate a real business cycle model. And the introduction of heterogeneity has predecessors in the literature. For comparability of our results, we aim at matching the same parameter combination.

We choose constant relative risk aversion as functional form for our utility specification defined over consumption. We set the coefficient of relative risk aversion to 2 . The time preference factor is chosen to be 0.95 .

We implement the penalty function for deviations from steady-state capital and bond holdings that imposes an endogenous borrowing constraint. Therefore, we set the parameter $\nu_{1}=3$ to ensure that the borrowing constraint receives a lot of weight. The parameter $\nu_{2}=0.01$ is set to a small number since it merely ensures that the steady-state is defined for the deterministic economy. Finally, we set $\nu_{3}=0.00001$ for the deterministic economy and to zero once we move to the stochastic version. To implement the borrowing constraint, we set $\underline{k}=-0.1$. As a consequence, for almost all cases, borrowing becomes prohibitively costly. The parameter $\bar{k}$ corresponds to the steady-state value of capital which is set such that the return on capital equals the reciprocal of the time preference factor in the deterministic steady-state.

The parameters governing the macreconomic considerations do not vary much across different works in the literature. We set the capital share of output to $\alpha$ to $1 / 3$ and the parameter for depreciation to 0.1 .

For the shocks to aggregate productivity and shocks to individual labor productivity, we follow the calibration in ? via ?. We set the stochastic process for aggregate uncertainty to be

$$
z_{t+1}=0.25+0.75 z_{t}+0.00661 \varepsilon_{t+1} .
$$

Idiosyncratic shocks to labor income evolve according to

$$
\psi_{t+1}^{i}=0.4+0.55555 \psi_{t}^{i}+\left(0.48989-0.28381 \psi_{t}^{i}\right) \theta_{t+1}^{i}
$$

We set upper and lower bounds for innovations to \pm 0.1 .

The question remaining question concerns the number of agents in the economy. As demonstrated when describing the solution method, the computing power required is the same for 
any number of individuals. To generate the results of this section, we set this number to 100 .

\subsection{Convergence}

As mentioned in section ??, the numerical method leads to a natural check of the accuracy of the solution. We normalize the Euler equation by dividing by marginal utility on both sides as in equation (??).

Figure ?? plots the logarithm of the Euler equation error as a function of one agent's capital stock. This check for accuracy corresponds to the deterministic version of the economy.



Figure 2: Euler equation error for the deterministic version of our economy.

The deterministic steady-state satisfies the deterministic optimality conditions. Thus the Euler equation error is zero at this point and its logarithm at negative infinity.

Two observations stand out from this graph. First, we see convergence. The Euler equation error decreases for the interval. And second, the result approximates the solution not just locally but globally on a sizeable interval.

\section{$5.3 \quad$ Impact of heterogeneity}

Heterogeneity with aggregate risk increases the steady-state level of capital. This result is known from the previous literature. There are two reasons for it. First, idiosyncratic risk leads to precautionary savings on the part of households. Since households cannot trade claims contingent on their labor income, they try to partially insure against these shocks by building up a buffer stock of savings. Second, due to aggregate productivity shocks, holding 
capital is risky. There are two opposing forces. On the one hand, agents are risk averse and demand a higher risk premium for holding risky capital. Each unit should thus return a higher dividend which implies a higher marginal product of capital and thus a lower steadystate level of capital. On the other hand though, since returns to capital are risky, agents again respond by building up savings which implies a higher steady-state level of capital. With our utility specification of constant relative risk aversion, the latter effect dominates. Thus, heterogeneity with aggregate risk increases the steady-state level of capital.

With respect to the pricing of assets, we study the impact of heterogeneity on the stochastic discount factor. We see that an aggregate stochastic discount factor defined as the average of all individual discount factors is approximated by

$$
\frac{\beta}{I} \sum_{i=1}^{I} \frac{u_{c}^{\prime}\left(c_{t+1}^{i}\right)}{u_{c}^{\prime}\left(c_{t}^{i}\right)}=c\left(\mathbf{X}_{t}, \mathbf{z}_{t}, \mathbf{X}_{t+1}\right)+\left(c_{z}^{(1)} e^{z_{t+1}}+c_{z}^{(2)} \operatorname{var}\left(e^{z_{t+1}}\right)+\ldots\right)+\left(c_{\psi}^{(2)} \operatorname{var}\left(\psi^{i}\right)+\ldots\right)
$$

The derivation of this expression can be found in appendix ??. The first term as well as all coefficients in the expansion are known at time $t$. The approximation thus tells us directly which assets demand a risk premium. Every security that comoves with total factor productivity, its variance (which we kept constant in our example), and higher moments will carry a risk premium.

But heterogeneity also enters the pricing of securities. The variance of individual labor income shocks is a risk factor. A time- or state-dependent variance will induce a risk premium for all assets that comove with this variance.

\section{Comparison between Methods for an Asset Pricing Problem}

We demonstrate the performance of our solution method in comparison with an approach that has been used frequently in the literature where you replace the law of motion by a linear function in the state variables.

To see the difference, we study a particularly simple asset pricing problem. ${ }^{6}$ We move to this particular economy because we obtain a closed-form solution which is not available for the previous example.

A representative agent prices a stochastic stream of endowments $C_{t}$ according to the following

\footnotetext{
${ }^{6}$ We borrow this problem from Stavros Panageas who introduced it to show that the linear law of motion can lead to erroneous results.
} 
stochastic process

$$
\log C_{t+1}=\log C_{t}+\mu+\varepsilon_{t+1}
$$

where the innovation $\varepsilon$ is distributed $\varepsilon \sim N\left(0, \sigma_{\varepsilon}^{2}\right)$. The representative agent's expected utility is given by the discounted stream of per-period utilities that feature constant relative risk aversion and two preference shocks $A$ and $B$

$$
\mathcal{U}_{t}=E_{t}\left[\sum_{t=0}^{\infty} \beta^{t} A_{t} B_{t} C_{t}^{1-\gamma}\right]
$$

The preference shocks evolve according to the stochastic processes

$$
\begin{aligned}
\log A_{t+1} & =\rho_{A} \log A_{t}+\sigma_{A} \eta_{t+1} \\
\log B_{t+1} & =\rho_{B} \log B_{t}+\sigma_{B} \eta_{t+1} .
\end{aligned}
$$

where the innovations are standard normally distributed.

\subsection{A closed-form solution}

To determine the value of the tree, we use the representative agent's Euler equation

$$
P_{0} A_{0} B_{0} C_{0}^{-\gamma}=\beta E_{t}\left[A_{1} B_{1} C_{1}^{-\gamma}\left(P_{1}+C_{1}\right)\right]
$$

and iterate forward to get

$$
\frac{P_{0}}{C_{0}}=\sum_{t=1}^{\infty} \beta^{t} e^{-\gamma \mu t+\frac{\gamma^{2} \sigma_{\varepsilon}^{2}}{2} t} A_{0}^{\rho_{A}^{t}-1} B_{0}^{\rho_{B}^{t}-1} e^{\frac{1}{2}\left[\sigma_{A}^{2} \frac{1-\rho_{A}^{2 t}}{1-\rho_{A}^{2}}+\sigma_{B}^{2} \frac{1-\rho_{B}^{2 t}}{1-\rho_{B}^{2}}+2 \sigma_{A} \sigma_{B} \frac{1-\rho_{A}^{t} \rho_{B}^{t}}{1-\rho_{A} \rho_{B}}\right]} .
$$

The derivation of this equation might not be immediate. Appendix ?? contains a derivation.

We can evaluate the quasi-closed-form solution with arbitrary accuracy by forward iteration given by equation (??). This reference solution serves as a benchmark for two approximation methods. First, we can assume a linear law of motion. Second, we can use the approximation method described in this paper to solve for the pricing. 


\subsection{Approximation with linear and quadratic laws of motion}

We re-write Euler equation (??) in terms of the price-dividend ratios

$$
\frac{P_{0}}{C_{0}}=E_{t}\left[\beta \frac{A_{1}}{A_{0}} \frac{B_{1}}{B_{0}}\left(\frac{C_{1}}{C_{0}}\right)^{1-\gamma}\left(\frac{P_{1}}{C_{1}}+1\right)\right] .
$$

A linear law of motion now describes the process for the price-dividend ratio

$$
\frac{P_{t+1}}{C_{t+1}}=\alpha+\rho_{p} \frac{P_{t}}{C_{t}}+\delta \eta_{t+1} .
$$

Using this linear law of motion, we arrive at a closed-form expression for the price dividend ratio given by

$$
\frac{P_{0}}{C_{0}}=\frac{\beta e^{-\gamma \mu+\frac{\gamma^{2} \sigma_{\varepsilon}^{2}}{2}} A_{0}^{\rho_{A}-1} B_{0}^{\rho_{B}-1} e^{\frac{1}{2}\left(\sigma_{A}+\sigma_{B}\right)^{2}}\left((1+\alpha)+\delta\left(\sigma_{A}+\sigma_{B}\right)\right)}{1-\rho_{p} \beta e^{-\gamma \mu+\frac{\gamma^{2} \sigma_{\varepsilon}^{2}}{2}} A_{0}^{\rho_{A}-1} B_{0}^{\rho_{B}-1} e^{\frac{1}{2}\left(\sigma_{A}+\sigma_{B}\right)^{2}}}
$$

Details on the derivation of this closed-form expression and on the quadratic law of motion are in appendix ??.

The coefficients in the linear law are chosen to maximize the fit with the dynamic evolution under this law of motion. Therefore, fix some coefficients, solve, and simulate the economy. Run a linear regression of next period's price-consumption ratio on this period's ratio. Use the resulting coefficients as a new law of motion and iterate until a fixed point is found.

\subsection{Approximation with our solution technique}

This problem features only a very mild level of heterogeneity. There are only two different taste shocks that enter the economy. The purpose of this section is to show that even for this mild level of heterogeneity, standard solution techniques might fail to provide an accurate solution.

We apply the solution method of this paper to Euler equation (??). The price-dividend ratio is a function of the two state variables $A_{t}$ and $B_{t}$. We start with the deterministic steady-state around which we approximate the price-dividend ratio. Then we procede in the standard fashion by building a high-order perturbation in the two state variables. Finally, we take the derivatives (and cross-derivatives with the two state variables) with respect to the standard deviation of the shocks. 
This is a particularly hard problem for the solution technique because the price-consumption ratios in the stochastic economy are in a different range from their deterministic counterparts which lies at a price consumption ratio of roughly 15 . If we set the standard deviations in equation (??) to zero, the deterministic price-consumption ration will be far smaller. In our later calibration, the stochastic price-consumption ratios will be more than ten times larger than the deterministic steady-state.

\subsection{Comparison}

We solve the economy for a particular parameter combination taken from Panageas. For this parameter combination, the $R^{2}$ criterion for the linear law of motion provides values above $98 \%$. Specifically, these paramters are a growth rate $\mu=1.4 \%$, risk aversion at $\gamma=8$, and the time discount factor $\beta=1.05$. The persistence of the two shocks is set to $\rho_{A}=0.98$ and $\rho_{B}=0.8$. The standard deviations are fixed at $\sigma_{\varepsilon}=0.04, \sigma_{A}=0.1$, and $\sigma_{B}=0.04$.
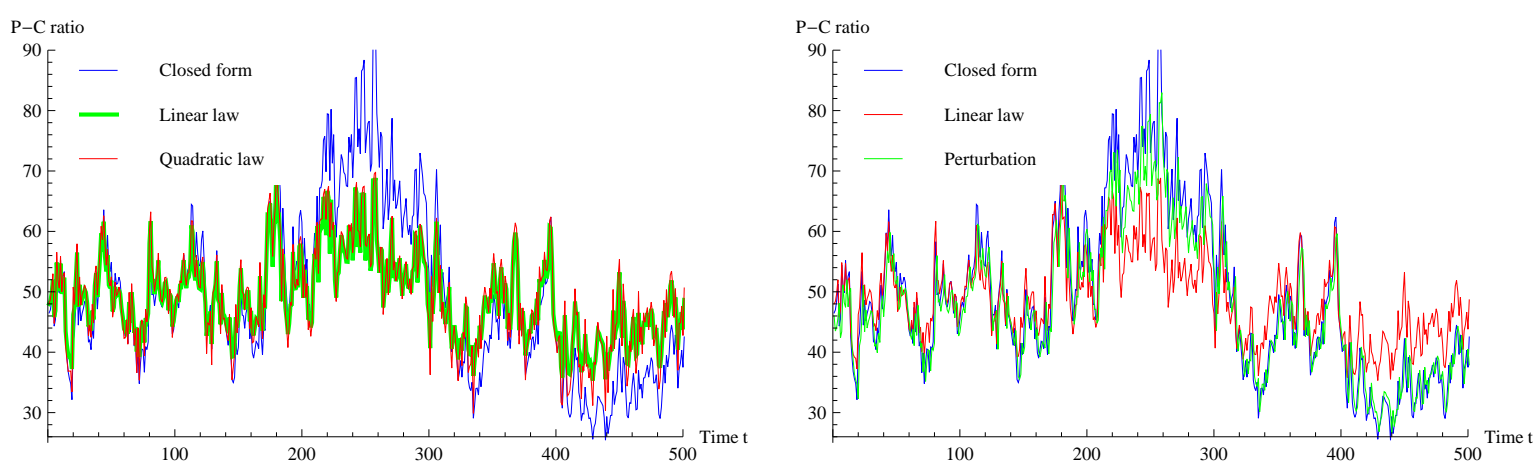

Figure 3: Comparison between the true solution and approximation methods using a linear law of motion and the perturbation approach of this paper.

Figure ?? compares the true solution to the approximations using a linear law of motion and the solution method of this paper. For the perturbation method, we choose an approximation of order 5 . We see that it fits the true solution closely while a linear law of motion deviates substantially although the $R^{2}$ diagnostic indicates a good fit. The implementation for the quadratic law of motion is numerically unstable the iteration only terminated when a high tolerance for the fixed point was chosen. Compared to the closed-form expression, the linear law of motion does not capture the variance of the time series.

This example was chosen to demonstrate difficulties when assuming a linear or quadratic law of motion. A linear/quadratic law of motion might deliver a poor approximation when the 
model is either highly nonlinear or when it is comprised of several state variables with different persistence. In the latter case, the sum of the state variables is not a good approximation to the joint distribution of the two variables.

\section{Conclusion}

In this paper, we presented the mathematical foundations for a class of incomplete market models with substantial heterogeneity. The analysis resulted in a proof of existence of equilibria both for small and large risks as well as a solution method. This method builds on perturbation methods which use Taylor series expansions around a deterministic steady-state. This solution method is particularly useful for models with many state and choice variables. Generally, this idea can be employed not only to competitive equilibria but also to dynamic programming problems. This feature suggests that the analysis can be applied to settings with recursive preferences. As a first example, we solved a dynamic stochastic general equilibrium model with idiosyncratic shocks to labor income. We demonstrated the convergence properties for this particular example. Furthermore, we showed that heterogeneity impacts macroeconomic quantities as well as the pricing of risk. 


\section{A Penalty Functions}

For an optimization problem of the form

$$
\max _{x} f(x) \text { subject to } c_{i}(x) \geq 0
$$

where $i$ serves as an index for constraints $c_{i}$, we can write a smooth version as

$$
\max _{x} f(x)-\mu \sum_{i} B\left(c_{i}(x)\right)
$$

where $B(\cdot)$ is continuously differentiable and $\lim _{x \rightarrow 0} B(x)=\infty$. Take a sequence of penalty parameters $\left\{\mu_{k}\right\}$ which leads to a sequence of solutions $\left\{x_{k}^{*}\right\}$ to (??). Then every limit point $x^{*}$ of the sequence of solutions $\left\{x_{k}^{*}\right\}$ is a global solution to the constrained optimization problem (??). For details, e.g. see ?.

\section{B Proofs for section ??}

\section{B.1 Construction of Compact Domain}

The compact domain $\Omega$ has to be chosen such that the equilibrium conditions are non-singular on this domain. For example, we need to rule out combinations of capital that lead aggregate capital to be negative while still allowing for short-selling of capital by individuals.

We define the domain through the boundaries in all directions in the 3I+1-dimensional space. To facilitate the definition of $\Omega$, we make it symmetric across individuals. Thus we only specify upper and lower bounds for the combination of state variables $k_{1}, k_{2}, b_{1}, b_{2}, \psi_{1}, \psi_{2}$, and $z$. All other restrictions follow by symmetry: for example, the restrictions on $k_{3}$ and $k_{4}$ are identical to those between $k_{1}$ and $k_{2}$.

We define restriction on shocks independent on where we are at the state space. We specify shocks to be on a compact support which increases with the perturbation variable $\sigma$. We thus calculate upper and lower bounds such that the shock in the following period always remains in the same domain. For the productivity shock, we achieve lower and upper bounds

$$
\underline{z}=\frac{1}{1-\phi_{z}} \underline{\varepsilon} \text { and } \bar{z}=\frac{1}{1-\phi_{z}} \bar{\varepsilon}
$$


Analogously, we obtain lower and upper bounds for individual labor income shocks via

$$
\underline{\psi}=\frac{\phi}{1-\phi_{\psi}}+\frac{\phi_{\theta}}{1-\phi_{\psi}} \underline{\theta} \text { and } \bar{\psi}=\frac{\phi}{1-\phi_{\psi}}+\frac{\phi_{\theta}}{1-\phi_{\psi}} \bar{\theta} .
$$

We specify the domain for the remaining variables $k_{1}, k_{2}, b_{1}$, and $b_{2}$ via the four combinations. After invoking symmetry, we are left with four upper and lower bounds. In the direction of $k_{1}$ and $k_{2}$, we impose

$$
\sum_{i=1}^{I} k_{i}>\underline{k}+\delta \text { and } \sum_{i=1}^{I} k_{i}<I \bar{k}
$$

The first constraint ensures positive levels of aggregate capital while allowing for short selling. The second level puts an upper bound on aggregate capital which is chosen large enough to ensure that at this point, agents chose to consume enough to decrease next period's capital stock.

Individual capital, however, has an upper bound given by the borrowing constraint

$$
k_{i}+b_{i}>\underline{k}+\delta \text { and } k_{i}+b_{i} \leq \bar{k}
$$

To pin down upper bounds on each variable, we use bounds such that the borrowing constraint cannot be violated even with zero consumption. We take

$$
\begin{aligned}
& f_{1}(I \bar{k}, L, \underline{\mathrm{z}}) k_{i} \geq f_{1}(I \underline{\mathrm{k}}, L, 1) b_{i}+e^{\psi} f_{2}(I \underline{\mathrm{k}}, L, \underline{\mathrm{z}}) \\
& -f_{1}(I \underline{\mathrm{k}}, L, \bar{z}) k_{i} \leq f_{1}(\bar{k}, L, 1) b+e^{\underline{\psi}} f_{2}(I \underline{\mathrm{k}}, L, \underline{\mathrm{z}})
\end{aligned}
$$

The first constraint ensures that long positions in capital do not lead to large violations of the endogenous borrowing constraint, the second is for short positions. Optimal choices will always obey these boundaries of the domain.

In the direction of bonds, we allow for small band

$$
-\delta \leq \sum_{i=1}^{I} b_{i} \leq \delta
$$

which is sufficient due to market clearing. The market clearing condition and the borrowing/leverage constraint also bound the domain in the $k_{i}-k_{j}$ direction. 


\section{B.2 Local Existence}

First, we need to make sure that a solution close to the deterministic steady-state stays within the same Banach space. Therefore, the solution cannot leave the domain $\Omega$ defined in ??. By construction, the shocks will remain within $\Omega$ for all $\sigma \in[0,1]$. For the deterministic economy, the solution is such that over time you converge towards the deterministic steady-state, i.e. it is asymptotically stable. For the stochastic economy, the penalty function imposes a force towards the steady-state. By choosing $\delta$ small enough, we can increase the domain so far that the endogenous borrowing constraint induces more saving and assures that we remain on the domain.

Second, the equilibrium operator $G(B(\sigma), \sigma)$ is differentiable at the deterministic steady-state since every first-order condition, budget constraint, and market clearing condition is differentiable on the domain. If we choose a slightly different choice or price function, the norm in the Banach space is bounded by the sum of the largest derivatives. Due to differentiability, this is a finite number. Thus we can derive the Fréchet derivative and the following lemma applies.

Lemma 1 If the operator $G$ lies in $\mathcal{C}^{m}$ as does the solution to the deterministic economy $\bar{B}$. Then we get, for a sufficiently small $r>0$ defining a ball $\mathcal{B}^{r}=\left\{B \in \mathcal{B}_{1}^{k} \mid\|B-\bar{B}\|_{\mathcal{B}_{1}^{k}}\right\}$ such that $G: \mathcal{B}^{r} \times[-\bar{\sigma}, \bar{\sigma}]$ for some $\bar{\sigma}$.

Proof: Due to the construction of the domain $\Omega$ and the fact that the deterministic solution is asymptotically stable, $G(B(\sigma), \sigma)$ exists and consumption is positive for all $\omega \in \Omega$. As long as operator and solution are part of $\mathcal{C}^{k}$, the order k derivatives of $G$ with respect to $\mathbf{X}, \mathbf{z}$, and $\sigma$ exist. Therefore, for a solution $B$ with $\|B-\bar{B}\|_{\mathcal{B}_{1}^{k}}$ sufficiently small, $G(B(\sigma), \sigma)$ exists and, by the chain rule, inherits differentiability.

Third, and last, we need invertibility of the derivative. We aim at a constructive proof and differentiate the equilibrium mapping with respect to $\sigma$ to arrive at the equation $G_{B}(B(0), 0) B_{\sigma}+$ $G_{\sigma}(B(0), 0)$. When solving for choices and prices, we realize that invertibility is a crucial property of equilibrium operators: $B_{\sigma}=-G_{B}(B(0), 0)^{-1} G_{\sigma}(B(0), 0)$. We aim at obtaining solutions of the form

$$
B(\sigma)=B(0)+\frac{\partial B}{\partial \sigma}(0) \sigma+\frac{1}{2} \frac{\partial^{2} B}{\partial \sigma^{2}}(0) \sigma^{2}+\ldots
$$

Now we study the effects of the first-order term.

Lemma 2 The deterministic solution does not solve any economy with small risks $\sigma \in$ 
$[-\bar{\sigma}, \bar{\sigma}]$

Proof: Plugging in the deterministic solution into the Euler equation with small risks leads to Euler equations of the form

$$
u^{\prime}\left(c_{t}^{i}\right)=\beta\left(1+r_{t}^{b}\right) E_{t}\left[u^{\prime}\left(c_{t+1}^{i}\right)\right]
$$

and

$$
u^{\prime}\left(c_{t}^{i}\right)=\beta E_{t}\left[1+r_{t}^{k}\right] E_{t}\left[u^{\prime}\left(c_{t+1}^{i}\right)\right]+\beta \operatorname{Cov}\left(1+r_{t}^{k}, u^{\prime}\left(c_{t+1}^{i}\right)\right) .
$$

Both equations cannot be satisfied simultaneously for the solution to the deterministic economy when the return to capital is risky.

Hence, the equilibrium operator produces locally separate solutions. For the deterministic economy, we had a unique solution. The operator being bijective implies invertibility of equilibrium conditions.

Lemma 3 The inverse operator $D G^{-1}(\mathbf{B}(0), 0)$ exists as a continuous linear operator if and only if the partial Fréchet derivative $D G(\mathbf{B}(0), 0)$ is bijective.

For a proof, see ?. Putting steps one to three together, we satisfy the conditions for the Implicit Function Theorem which guarantees local existence of equilibria.

\section{B.3 Proof of Proposition ??}

To prove the proposition, we first revisit the underlying structure behind the Leray-Schauder continuation theorem. The previous section dealt with the local nature of the problem using the Implicit Function Theorem. To analyze the global nature, we revisit degree theory. A degree indicates whether a function has a solution to the equation $f(\omega)=0$ on a certain domain $\Omega$ with a boundary $\partial \Omega$. We start by defining the degree for finite-dimensional maps from $\Omega \subset \mathbb{R}^{N}$ to $\mathbb{R}^{N}$. For a mapping $f$ which satisfies the properties

(1) $0 \notin f(\partial \Omega)$

(2) $f$ is continuously differentiable on $\Omega$

(3) If $\omega \in \Omega$ such that $f(\omega)=0$, then $\operatorname{det} D f(\omega) \neq 0$. 
For these mappings, one can define the Brower degree via

$$
d(f, \Omega, 0)=\sum_{\omega \in f^{-1}(0)} \operatorname{sign} \operatorname{det} D f(\omega)
$$

As a result, if the degree takes on a value of -1 , it means that the mapping crossed zero at least once from a positive value to a negative value over the domain. A value of zero indicates no or an equal number of crossings and a value of one suggests one or an uneven number of crossings from negative to positive. The Brouwer degree has several useful properties:

Normalization The degree of the identity map $I$ is

$$
d(I, \Omega, 0)= \begin{cases}1, & 0 \in \Omega \\ 0, & 0 \notin \Omega\end{cases}
$$

Solution Property If $d(f, \Omega, 0) \neq 0$, then there exists $\omega \in \Omega$ such that $f(\omega)=0$.

Homotopy Invariance If $H:[0,1] \times \bar{\Omega} \rightarrow \mathbb{R}^{N}$ is continuous, with $H(\sigma, \omega) \neq 0$ for all $\omega \in \partial \Omega$ and $\sigma \in[0,1]$, then $d(H(\sigma, \cdot), \Omega, 0)$ is constant (i.e. independent of $\sigma$ ).

Excision If $U$ is a closed subset of $\bar{\Omega}$ and $f$ has no zeros in $U$, then $d(f, \Omega, 0)=d(f, \Omega \backslash U, 0)$.

As described in ?, the definition of Brouwer degrees allows for a direct proof of the Brouwer Fixed Point Theorem which allows to show the existence of solutions.

Schauder achieved a generalization of the Brouwer degree to infinite-dimensionsal mappings. The difficulty lies in the fact that the closed unit ball in infinite-dimensional spaces is not compact and will, in general, not have a fixed point property. The important ingredient in making progress is some kind of compactness in Banach spaces $\mathcal{B}$.

\section{Definition 5 (Compact mapping)}

$A$ mapping $G: \mathcal{B} \rightarrow \mathcal{B}$ is compact if for each bounded set $B \subset \mathcal{B}$, the set $\overline{G(B)}$ is compact.

Using compactness, we define complete continuity which is one of the pillars of the LeraySchauder principle.

\section{Definition 6 (Completely continuous)}

A mapping $G: \mathcal{B} \rightarrow \mathcal{B}$ is completely continuous if it is compact and continuous. 


\section{Definition 7 (Completely continuous perturbation of the identity)}

A mapping of the form $\hat{G}=I-G$, where $G$ is completely continuous is called a completely continuous perturbation of the identity.

It turns out that compact sets in Banach spaces are nearly finite dimensional in the sense that one can approximate compact sets with finite dimensional sets.

\section{Lemma 4 (Schauder projection)}

Let $\mathcal{K}$ be a compact subset of the Banach space $\mathcal{B}$. Given $\varepsilon>0$, there exists a finite dimensional convex set $\mathcal{Y} \subset \mathcal{B}$ and a map $P_{\varepsilon}: \mathcal{K} \rightarrow \mathcal{Y}$ such that

$$
\left\|P_{\varepsilon}(x)-x\right\|<\varepsilon \quad \text { for all } x \in \mathcal{K}
$$

The map $P_{\varepsilon}$ is called Schauder projection.

Since the Schauder projection is arbitrarily close to the identity, compact subsets can be shown to be nearly finite-dimensional. For completely continuous mappings, the Brouwer degree can be generalized as the Leray-Schauder degree. Then for every completely continuous map $G: \bar{\Omega} \rightarrow \mathcal{B}$ such that $\omega-G(\omega) \neq 0$ for all $\omega \in \partial \Omega$, there exists a unique integer $d(I-G, \Omega, 0)$ with similar properties to the ones above.

Normalization The degree of the identity map $I$ is

$$
d(I, \Omega, 0)= \begin{cases}1, & 0 \in \Omega \\ 0, & 0 \notin \Omega\end{cases}
$$

Solution Property If $d(I-G, \Omega, 0) \neq 0$, then there exists $\omega \in \Omega$ such that $G(\omega)=\omega$.

Homotopy Invariance If $H:[0,1] \times \bar{\Omega} \rightarrow \mathbb{R}^{N}$ is completely continuous, with $\omega \neq H(\sigma, \omega)$ for all $\omega \in \partial \Omega$ and $\sigma \in[0,1]$, then $d(H(\sigma, \cdot), \Omega, 0)$ is constant.

Excision If $K$ is a closed subset of $\bar{\Omega}$ and $F$ has no fixed points in $K$, then $d(I-G, \Omega, 0)=$ $d(I-G, \Omega \backslash K, 0)$.

\section{Theorem 3 (Schauder fixed point theorem)}

If $K$ is a closed, bounded, and convex subset of $\mathcal{B}$ and $G: K \rightarrow K$ is completely continuous, then $G$ has a fixed point in $K$.

Leray and Schauder developed a method to continue solutions based on the ideas underlying the fixed point theorem. Therefore, we let $\mathcal{O}$ denote a bounded open subset of $\mathcal{B}_{1} \times[\underline{\sigma}, \bar{\sigma}]$ 
and let $\mathcal{O}_{\sigma}=\left\{\mathbf{B} \in \mathcal{B}_{1}:(\mathbf{B}, \sigma) \in \mathcal{O}\right\}$ denote the projection onto $\mathcal{B}_{1}$. We define $\hat{G}$ by

$$
\hat{G}(\mathbf{B}, \sigma)=\mathbf{B}-G(\mathbf{B}, \sigma)
$$

where $G: \bar{U} \rightarrow \mathcal{B}_{1}$ is completely continuous and

$$
G(\mathbf{B}, \sigma) \neq 0 \text { for all } \sigma \in[\underline{\sigma}, \bar{\sigma}] \text { and } \mathbf{B} \in \partial \mathcal{O} \text {. }
$$

Then the following lemma holds.

\section{Lemma 5 (Generalized Homotopy)}

If $\hat{G}$ is defined by (??) and satisfies (??), then $d\left(\hat{G}(\cdot, \sigma), \mathcal{O}_{\sigma}, 0\right)$ is independent of $\sigma$.

\section{Theorem 4 (Leray-Schauder Continuation)}

Let $\hat{G}$ be defined by (??) and satisfy (??). If $d\left(\hat{G}, \mathcal{O}_{\underline{\sigma}}, 0\right) \neq 0$, then there exists a continuum $\mathcal{C}$ such that $\mathcal{C} \cap \mathcal{O}_{\underline{\sigma}} \neq \emptyset \neq \mathcal{C} \cap \mathcal{O}_{\bar{\sigma}}$.

The Leray-Schauder continuation theorem gives conditions under which we can continue the local solution to the case of larger risk. Therefore, we need to make sure that the domain is large enough for the solution to map elements of the domain into it. Hence we set $\varepsilon_{1}>0$ small enough in the definition of the domain $\Omega$. We define the compact interval for the solution as $\underline{\mathbf{k}}=\varepsilon_{1}$ and $\overline{\mathbf{k}}=2 \overline{\mathbf{k}}^{*}-\varepsilon_{1}$ where $\overline{\mathbf{k}}^{*}$ denotes the amount of capital in the symmetric deterministic steady-state. Then we choose $\varepsilon_{2} \ll \varepsilon_{1}$ to define the subset of our Banach space

$$
B_{p}=\left\{(\mathbf{c}, \mathbf{k}) \mid c_{i}(\mathbf{k}, \psi, \mathbf{z}, \sigma) \geq \varepsilon_{2} \quad \forall \mathbf{k}, \psi, \mathbf{z}, \sigma \quad \forall i \text { and }\|\bar{c}\| \leq 2 f\left(2 k^{*}, l\right)\right\}
$$

This allows us to define a subset of the space $\mathcal{B}_{1}$ via $B=\mathbb{I} \times B_{p}$.

The major step in the proof of the proposition remains to show that the operator $G$ is completely continuous on the subset $B$. Therefore, we need to show that, taken an arbitrary bounded subset of $B$, the image under the operator $G(B)$ is compact, i.e. the closure of the image is compact.

\section{Lemma 6 (Boundedness of the operator)}

$A$ bounded subset $B \subset \Omega$ has a bounded image under the operator $\hat{G}$.

Proof: To get an upper and lower bound, we go to the boundary of $B$ and map it using the operator $\hat{G}$. We know that marginal utility will be high and the discrepancy from 0 the highest if we set consumption to its lowest level. However, since we are bounded away from zero, we will have a bounded image. 


\section{Lemma 7 (Convergence of sequences)}

An arbitrary bounded sequence in $B$ is mapped into a sequence $\left\{y_{n}\right\}_{n \in \mathbb{N}}$ in $G(B)$. The sequence $\left\{y_{n}\right\}_{n \in \mathbb{N}}$ has a convergent subsequence.

Proof: Since the operator $G$ maps into a Banach space, we can measure the norm of the sequence. Our image $G(B)$ is bounded and so will be the norms of all the elements in the image. Since the sequence $\left\{\left\|G_{\sigma}\right\|\right\}_{n \in \mathbb{N}}$ is bounded as well, we can find a subsequence whose norm converges. Take that subsequence. Its norms converge in $\mathcal{B}_{2}$ and the limiting element will lie in the closure of the image.

From the previous two lemmas, we get that the image is compact which implies that $G$ is completely continuous on $B$. We now need to establish the behavior of the operator on the boundary.

\section{Lemma 8 (Absence of solutions on the boundary)}

The operator $G$ does not possess solutions on the boundary of $B \times[0,1]$, i.e.

$$
G(\omega, \sigma) \neq 0 \quad \forall \omega \in \partial \Omega .
$$

Proof: We set up the set $B$ in a way that agents either overconsume on one side of the boundary and underconsume on the other. On one side of the boundary, consumption is very close to zero for all levels of capital and on the other side of the boundary consumption equals or exceeds wealth. These elements are suboptimal and do not represent solutions.

We are now in the position to apply the Leray Schauder continuation theorem. Thus we can now transform the deterministic solution into a solution for the large case of risk. 


\section{Approximation of the Law of Motion}

\section{C.1 The Linear Law of Motion}

Next period's average capital as $\frac{1}{I} K_{t+1}=\frac{1}{I} \sum_{i=1}^{I} k_{t+1}^{i}\left(\mathbf{X}_{t}, \mathbf{z}_{t}, \sigma\right)$. Using equation (??), we get a first-order approximation of the form

$$
\begin{aligned}
\frac{1}{I} K_{t+1} \approx & k_{0} \\
& +\sum_{i=1}^{I} k_{\mathbf{X}_{11}}^{i}\left(\mathbf{X}_{11}-\overline{\mathbf{X}}_{11}\right)+k_{\mathbf{X}_{21}}^{i}\left(\mathbf{X}_{21}-\overline{\mathbf{X}}_{21}\right)+k_{\mathbf{X}_{31}}^{i}\left(\mathbf{X}_{31}-\overline{\mathbf{X}}_{31}\right)+k_{\mathbf{X}_{41}}^{i}\left(\mathbf{X}_{41}-\overline{\mathbf{X}}_{41}\right)+\ldots \\
& +\sum_{i=1}^{I} k_{\mathbf{X}_{12}}^{i}\left(\mathbf{X}_{12}-\overline{\mathbf{X}}_{12}\right)+k_{\mathbf{X}_{22}}^{i}\left(\mathbf{X}_{22}-\overline{\mathbf{X}}_{22}\right)+k_{\mathbf{X}_{32}}^{i}\left(\mathbf{X}_{32}-\overline{\mathbf{X}}_{32}\right)+k_{\mathbf{X}_{42}}^{i}\left(\mathbf{X}_{42}-\overline{\mathbf{X}}_{42}\right)+\ldots \\
& +\ldots \\
& +\sum_{i=1}^{I} k_{\mathbf{z}_{1}}^{i}\left(\mathbf{z}_{1}-\overline{\mathbf{z}}_{1}\right)+k_{\mathbf{z}_{2}}^{i}\left(\mathbf{z}_{2}-\overline{\mathbf{z}}_{2}\right)+k_{\mathbf{z}_{3}}^{i}\left(\mathbf{z}_{3}-\overline{\mathbf{z}}_{3}\right)+\ldots
\end{aligned}
$$

where $\overline{\mathbf{X}}_{\cdot 1}$ is the deterministic steady-state value.

With the assumptions on symmetry, coefficients on expansions as well as steady-state values are identical and summarize to

$$
\begin{aligned}
\frac{1}{I} K_{t+1} \approx & k_{0} \\
& +\left(k_{\mathbf{X}_{11}}^{1}-k_{\mathbf{X}_{21}}^{1}\right)\left(\mathbf{X}_{1}-\overline{\mathbf{X}}_{11}\right)+I k_{\mathbf{X}_{21}}^{i}\left(\mathbf{X}_{1}-\overline{\mathbf{X}}_{11}\right) \\
& +\left(k_{\mathbf{X}_{12}}^{1}-k_{\mathbf{X}_{22}}^{1}\right)\left(\mathbf{X}_{2}-\overline{\mathbf{X}}_{12}\right)+I k_{\mathbf{X}_{22}}^{i}\left(\mathbf{X}_{2}-\overline{\mathbf{X}}_{12}\right) \\
& +\sum_{i=1}^{I} k_{\mathbf{z}_{1}}^{i}\left(\mathbf{z}_{1}-\overline{\mathbf{z}}_{1}\right)+k_{\mathbf{z}_{2}}^{i}\left(\mathbf{z}_{2}-\overline{\mathbf{z}}_{2}\right)+k_{\mathbf{z}_{3}}^{i}\left(\mathbf{z}_{3}-\overline{\mathbf{z}}_{3}\right)+\ldots
\end{aligned}
$$

where $\mathbf{X}_{j}=\frac{1}{I} \sum_{i=1}^{I} \mathbf{X}_{i j}$

\section{C.2 The Quadratic Law of Motion}

For the second-order terms, we again build an expansion for one policy and sum up over all agents. Thereby, we invoke symmetry in the analogous fashion. Simply regrouping the terms 
from equation (??)

$$
\begin{aligned}
k^{1}(X, z) \approx & k_{0}+\text { first-order terms } \\
& +\left(k_{\mathbf{X}_{11}, \mathbf{X}_{11}}^{1}-k_{\mathbf{X}_{21}, \mathbf{X}_{21}}^{1}-2 k_{\mathbf{X}_{11}, \mathbf{X}_{21}}^{1}+k_{\mathbf{X}_{21}, \mathbf{X}_{31}}^{1}\right)\left(\mathbf{X}_{11}-\overline{\mathbf{X}}_{1}\right)^{2} \\
& +\left(k_{\mathbf{X}_{21}, \mathbf{X}_{21}}^{1}-k_{\mathbf{X}_{21}, \mathbf{X}_{31}}^{1}\right) \sum_{i=1}^{I}\left(\mathbf{X}_{i 1}-\overline{\mathbf{X}}_{1}\right)^{2} \\
& +\left(2 k_{\mathbf{X}_{11}, \mathbf{X}_{21}}^{1}-k_{\mathbf{X}_{21}, \mathbf{X}_{31}}^{1}\right)\left(\mathbf{X}_{11}-\overline{\mathbf{X}}_{1}\right)\left(\mathbf{X}_{1}-\overline{\mathbf{X}}_{1}\right) \\
& +k_{\mathbf{X}_{21}, \mathbf{X}_{31}}^{1}\left(\mathbf{X}_{1}-\overline{\mathbf{X}}_{1}\right)^{2}
\end{aligned}
$$

To get to average capital, we average across all agents and invoke symmetry

$$
\begin{aligned}
& \frac{1}{I} \sum_{i=1}^{I} k^{i}(X, z) \approx \\
& \qquad k_{0}+\text { first-order terms } \\
& +\left(k_{\mathbf{X}_{11}, \mathbf{X}_{11}}^{1}-2 k_{\mathbf{X}_{11}, \mathbf{X}_{21}}^{1}\right) \sum_{i=1}^{I}\left(\mathbf{X}_{i 1}-\overline{\mathbf{X}}_{1}\right)^{2}+2 k_{\mathbf{X}_{11}, \mathbf{X}_{21}}^{1}\left(\mathbf{X}_{1}-\overline{\mathbf{X}}_{1}\right)^{2}
\end{aligned}
$$

\section{C.3 Approximation of any symmetric variable}

The previous logic goes through for every approximation of a variable $f(\mathbf{k})$ where

$$
\frac{\partial f}{\partial k^{i}}=\frac{\partial f}{\partial k^{j}} \quad \forall i, j
$$

The first-order expansion

$$
f(\mathbf{X}) \approx f(\overline{\mathbf{X}})+f_{\mathbf{X}_{11}}(\overline{\mathbf{X}})\left(\mathbf{X}_{1}-\overline{\mathbf{X}}_{1}\right)+f_{\mathbf{X}_{21}}(\overline{\mathbf{X}})\left(\mathbf{X}_{2}-\overline{\mathbf{X}}_{2}\right)+\ldots
$$

The symmetry conditions are simply

(i) $\frac{d^{2} f}{d x_{i}^{2}}=\frac{d^{2} f}{d x_{1}^{2}}$

(ii) $\frac{d^{2} f}{d x_{i} d x_{j}}=\frac{d^{2} f}{d x_{1} d x_{2}}$ for $i \neq j$

Using these conditions, we can simplify the expansion of $f$ analogously to before. We get

$$
f(\mathbf{X}) \approx f_{0}+\text { first-order terms }+I\left(\frac{d^{2} f}{d x_{1}^{2}}-\frac{d^{2} f}{d x_{1} d x_{2}}+I^{2} \frac{d^{2} f}{d x_{1} d x_{2}}\right)\left(X_{1}-\bar{X}_{1}\right)^{2}
$$




\section{Approximation of a Stochastic Discount Factor}

One stochastic discount factor is given by the average individual stochastic discount factor. We use the technique of a nonlinear change of variables to approximate it with pertubation methods. Therefore, we recognize that the marginal utility of next period's consumption is a function of $\mathbf{X}_{t+1}$ and $\mathbf{z}_{t+1}$ while the marginal utility of consumption today is a function of today's state variables. Together, we build one Taylor expansion with respect to all these state variables. Applying the logic from equation (??), we arrive at

$$
\frac{\beta}{I} \sum_{i=1}^{I} \frac{u^{\prime}\left(c_{t+1}^{i}\right)}{u^{\prime}\left(c_{t}^{i}\right.} \approx \sum_{o=0}^{\infty} \sum_{\|\mathbf{I}\|+|\mathbf{j}|+k=o} \frac{1}{\mathbf{I} ! \cdot \mathbf{j} ! \cdot k !}\left(h_{t, \mathbf{I}, \mathbf{j}, k}\left(\mathbf{X}_{t}, \mathbf{z}_{t}\right)+h_{t+1, \mathbf{I}, \mathbf{j}, k}\left(\mathbf{X}_{t+1}, \mathbf{z}_{t+1}\right)\right)
$$

where $h_{,, \mathbf{I}, \mathbf{j}, k}\left(\mathbf{X}_{t}, \mathbf{z}_{t}\right)=\mathbf{U}^{(\mathbf{I}, \mathbf{j}, k)}\left(\mathbf{X}^{0}, \mathbf{z}^{0}, 0\right)\left(\mathbf{X}-\mathbf{X}^{0}\right)^{\mathbf{I}}\left(\mathbf{z} .-\mathbf{z}^{0}\right)^{\mathbf{j}} \sigma^{k}$. The function $U$ is represents marginal utility of consumption for period $t+1$ and the inverse thereof for period $t$. The derivatives at the deterministic steady-state are computed using the nonlinear change of variables.

Given this expansion, collect all monomial terms merely depending on state variables known in period $t$, i.e. $\mathbf{X}_{t}, \mathbf{z}_{t}$, and $\mathbf{X}_{t+1}$. The collection of those terms is denoted by $c\left(\mathbf{X}_{t}, \mathbf{z}_{t}, \mathbf{X}_{t+1}\right)$ in equation (??). Next, collect all terms in which total factor productivity appears linearly. These terms are the first-order term in the expansion of productivity and all cross-terms with variables known at time $t$. Collect those in a term $c_{z}^{(1)}\left(\mathbf{X}_{t}, \mathbf{z}_{t}, \mathbf{X}_{t+1}\right)$ where we drop the arguments in equation (??). We similarly collect the terms for second-order expansions with respect to total factor and individual productivity and arrive at equation (??).

\section{E Asset Pricing Example - Derivations}

\section{E.1 Derivation of the Closed Form Solution for the Asset Pricing Economy}

We start from the Euler equation of the tree (??) which we rewrite in the following form

$$
\frac{P_{0}}{C_{0}}=E_{t}\left[\beta \frac{A_{1}}{A_{0}} \frac{B_{1}}{B_{0}}\left(\frac{C_{1}}{C_{0}}\right)^{1-\gamma}\left(\frac{P_{1}}{C_{1}}+1\right)\right]
$$

and iterate to get

$$
\frac{P_{0}}{C_{0}}=E_{t}\left[\sum_{t=1}^{\infty} \beta^{t} \frac{A_{t}}{A_{0}} \frac{B_{t}}{B_{0}}\left(\frac{C_{t}}{C_{0}}\right)^{1-\gamma}\right]
$$


Now we plug in the stochastic processes which we iterate to get

$$
\frac{A_{t}}{A_{0}}=A_{0}^{\rho_{A}^{t}-1} e^{\sigma_{A} \sum_{j=0}^{t-1} \rho_{A}^{j} \eta_{t-j}}
$$

and

$$
\frac{B_{t}}{B_{0}}=B_{0}^{\rho_{B}^{t}-1} e^{\sigma_{B} \sum_{j=0}^{t-1} \rho_{B}^{j} \eta_{t-j}} .
$$

As a result, the expectation over the product of these ratios reads

$$
\begin{aligned}
E_{t}\left[\frac{A_{t}}{A_{0}} \frac{B_{t}}{B_{0}}\right]= & A_{0}^{\rho_{A}^{t}-1} B_{0}^{\rho_{B}^{t}-1} e^{\sum_{j=0}^{t-1}\left(\sigma_{A} \rho_{A}^{j}+\sigma_{B} \rho_{B}^{j}\right) \eta_{t-j}} \\
& =A_{0}^{\rho_{A}^{t}-1} B_{0}^{\rho_{B}^{t}-1} e^{\frac{1}{2}\left[\sigma_{A}^{2} \frac{1-\rho_{A}^{2 t}}{1-\rho_{A}^{2}}+\sigma_{B}^{2} \frac{1-\rho_{B}^{2 t}}{1-\rho_{B}^{2}}+2 \sigma_{A} \sigma_{B} \frac{1-\rho_{A}^{t} \rho_{B}^{t}}{1-\rho_{A} \rho_{B}}\right]} .
\end{aligned}
$$

We plug this equation in our iterated Euler equation

$$
\begin{aligned}
\frac{P_{0}}{C_{0}} & =\sum_{t=1}^{\infty} E_{t}\left[\beta^{t} \frac{C_{t}^{1-\gamma}}{C_{0}^{1-\gamma}}\right] \cdot E_{t}\left[\frac{A_{t}}{A_{0}} \frac{B_{t}}{B_{0}}\right] \\
& =\sum_{t=1}^{\infty} \beta^{t} e^{-\gamma \mu t+\frac{\gamma^{2} \sigma_{\varepsilon}^{2}}{2} t} A_{0}^{\rho_{A}^{t}-1} B_{0}^{\rho_{B}^{t}-1} e^{\frac{1}{2}\left[\sigma_{A}^{2} \frac{1-\rho_{A}^{2 t}}{1-\rho_{A}^{2}}+\sigma_{B}^{2} \frac{1-\rho_{B}^{2}}{1-\rho_{B}^{2}}+2 \sigma_{A} \sigma_{B} \frac{1-\rho_{A}^{t} \rho_{B}^{t}}{1-\rho_{A} \rho_{B}}\right]}
\end{aligned}
$$

which yields our result in equation (??).

\section{E.2 Linear Law of Motion}

From the linear law of motion (??) and the Euler equation (??), we receive an equation

$$
\frac{P_{0}}{C_{0}}=E_{0}\left[\beta \frac{A_{1}}{A_{0}} \frac{B_{1}}{B_{0}}\left(\frac{C_{1}}{C_{0}}\right)^{1-\gamma}\left(1+\alpha+\rho_{p} \frac{P_{0}}{C_{0}}+\delta \eta_{1}\right)\right]
$$

that we need to solve. We rearrange it to

$$
\frac{P_{0}}{C_{0}}=\frac{\beta e^{-\gamma \mu+\frac{\gamma^{2} \sigma_{\varepsilon}^{2}}{2}} E_{0}\left[\frac{A_{1}}{A_{0}} \frac{B_{1}}{B_{0}}\left(1+\alpha+\delta \eta_{1}\right)\right]}{1-\rho_{p} \beta e^{-\gamma \mu+\frac{\gamma^{2} \sigma_{\varepsilon}^{2}}{2}}}
$$

and solve for the different parts. First note that

$$
E_{0}\left[\frac{A_{1}}{A_{0}} \frac{B_{1}}{B_{0}}\right]=A_{t}^{\rho_{A}-1} B_{t}^{\rho_{B}-1} e^{\frac{1}{2}\left(\sigma_{A}+\sigma_{B}\right)^{2}}
$$


which simplifies the denominator. For the numerator, we make use of the fact that consumption growth and growth of taste shocks are independent. Thus, we can treat the terms in the expectation separately. For the preference shocks, we get

$$
E_{1}\left[\frac{A_{1}}{A_{0}} \frac{B_{1}}{B_{0}}\left(1+\alpha+\delta e^{\eta_{1}}\right)\right]=A_{1}^{\rho_{A}-1} B_{1}^{\rho_{B}-1}\left(e^{\frac{1}{2}\left(\sigma_{A}+\sigma_{B}\right)^{2}}(1+\alpha)+\delta\left(\sigma_{A}+\sigma_{B}\right) e^{\frac{\left(\sigma_{A}+\sigma_{B}\right)^{2}}{2}}\right)
$$

where the first part comes from a standard iteration as before. The second part follows from

$$
E_{1}\left[\frac{A_{1}}{A_{0}} \frac{B_{1}}{B_{0}} \delta \eta_{1}\right]=A_{0}^{\rho_{A}-1} B_{0}^{\rho_{B}-1} \delta E_{0}\left[e^{\left(\sigma_{A}+\sigma_{B}\right) \eta_{1}} \eta_{1}\right] .
$$

The last expectation can be computed by solving the integral

$$
\begin{aligned}
\int_{-\infty}^{\infty} \frac{1}{\sqrt{2 \pi}} x e^{-\frac{1}{2}\left(x-\left(\sigma_{A}+\sigma_{B}\right)\right)^{2}} e^{-\frac{x^{2}}{2}} d x & =e^{\frac{\left(\sigma_{A}+\sigma_{B}\right)^{2}}{2}} \int_{-\infty}^{\infty} \frac{1}{\sqrt{2 \pi}} x e^{-\frac{1}{2}\left(x-\left(\sigma_{A}+\sigma_{B}\right)\right)^{2}} d x \\
& =\left(\sigma_{A}+\sigma_{B}\right) e^{\frac{\left(\sigma_{A}+\sigma_{B}\right)^{2}}{2}}
\end{aligned}
$$

\section{E.3 Quadratic law of motion}

Agents perceive the equation of motion to be

$$
\frac{P_{t+1}}{C_{t+1}}=\alpha+\rho_{p 1} \frac{P_{t}}{C_{t}}+\rho_{p 2}\left(\frac{P_{t}}{C_{t}}\right)^{2}+\delta \eta_{t+1}
$$

Plug the equation of motion into the Euler equation to get

$$
\frac{P_{t}}{C_{t}}=E_{t}\left[\beta \frac{A_{t+1}}{A_{t}} \frac{B_{t+1}}{B_{t}}\left(\frac{C_{t+1}}{C_{t}}\right)^{1-\gamma}\left(\alpha+\rho_{p 1} \frac{P_{t}}{C_{t}}+\rho_{p 2}\left(\frac{P_{t}}{C_{t}}\right)^{2}+\delta \eta_{t+1}+1\right)\right]
$$

or simplified as

$$
\Psi_{t}(\alpha+1)+\left(\rho_{p 1} \Psi_{t}-1\right) \frac{P_{t}}{C_{t}}+\Psi_{t} \rho_{p 1}\left(\frac{P_{t}}{C_{t}}\right)^{2}+E_{t}\left[\beta \frac{A_{t+1}}{A_{t}} \frac{B_{t+1}}{B_{t}}\left(\frac{C_{t+1}}{C_{t}}\right)^{1-\gamma} \delta \eta_{t+1}\right]=0
$$

where

$$
\Psi_{t}=E_{t}\left[\beta \frac{A_{t+1}}{A_{t}} \frac{B_{t+1}}{B_{t}}\left(\frac{C_{t+1}}{C_{t}}\right)^{1-\gamma}\right]
$$

The formula for quadratic equations delivers two solutions, one of which is the desired one. 